\title{
Understanding the physics of the auxetic response in a liquid crystal elastomer
}

\author{
Thomas Raistrick ๑, ${ }^{*}$ Zhaopeng Zhang, Devesh Mistry ๑, Johan Mattsson ๑, and Helen F. Gleeson $\odot$ \\ School of Physics and Astronomy, University of Leeds, Leeds, LS2 9JT, United Kingdom
}

(Received 23 June 2020; accepted 20 May 2021; published 8 June 2021)

\begin{abstract}
Synthetic materials that are auxetic (negative Poisson's ratio) at a molecular level have long been sought after. An auxetic liquid crystal elastomer (LCE) was reported recently, but the physical origin of the behavior has remained unclear. Here, we explain the physics behind the auxetic response of a uniaxial LCE under strain by the emergence of biaxial order. Our study relies on understanding order parameters (OPs), determined via Raman scattering, for LCEs strained either parallel or perpendicular to the director and explaining their relevance in terms of existing LCE theory. In particular, we consider a 3D interpretation of the orientational distribution function. We use uniaxial and biaxial models to deduce that (i) a changing nematic order drives the mechanical Fréedericksz transition (MFT), (ii) the apparent semi-soft elasticity exhibited by this LCE is related to this change in order, (iii) there is also an emergence of biaxial order in the LCE, and (iv) the emergence of biaxiality explains the auxetic response. Uniaxial OPs, $\left\langle P_{200}\right\rangle$ and $\left\langle P_{400}\right\rangle$, initially take values of 0.6 and 0.3 and, for strains parallel to the director, increase by $20 \%$. For perpendicular strains, the OPs reduce dramatically with increasing strain, becoming zero at the MFT, where the director reorients to align with the strain axis; the auxetic behavior emerges in the same strain regime. Our explanation identifies key parameters in determining the requirements for auxetic behavior in LCEs.
\end{abstract}

DOI: 10.1103/PhysRevResearch.3.023191

\section{INTRODUCTION}

Liquid crystalline elastomers (LCEs) are a fascinating class of materials which combine the fields of liquid crystals and polymers. LCEs are crosslinked polymers that include liquid crystalline moieties substituted as side-chain units, mainchain units, or both. They are perhaps best known for some of their remarkable properties, such as stress-optical coupling and stimuli responsiveness, that lend themselves to innovative applications including photo-actuated microrobots, chemical sensors, substrates for flexible electronics, and variable irises, to name but a few [1-4]. Recently, some LCEs have been found to display an auxetic response (negative Poisson's ratio) on the molecular scale, making these the first of their kind [5]. While examples of natural molecular auxetics exist [6], a synthetic auxetic response is typically achieved via deformation of structural geometries such as honeycomblike structures and porous structures, almost all of which are large scale ( $>10$ s of microns) [7-10]. An elegant example of a nanoscale structure is reported by Suzuki et al. [11] where self-assembled hinged nanocrystals are viewed by transmission electron microscopy to display an auxetic response with a Poisson's Ratio of -1 . The auxetic behavior occurs through reorientation of porous crystal lattice structures, with crystal sizes limited to microns.

\footnotetext{
*py14tr@leeds.ac.uk
}

Published by the American Physical Society under the terms of the Creative Commons Attribution 4.0 International license. Further distribution of this work must maintain attribution to the author(s) and the published article's title, journal citation, and DOI.
Many other attempts have been made to synthesise molecular auxetic materials [12-14], but the closest materials to those we describe in this paper are designs involving liquid crystalline polymers $[15,16]$. Of particular note is the synthesis of a main-chain liquid crystalline polymer with alternating terminally and laterally attached mesogenic units along the chain [16]. It was suggested that stretching the material would cause the rotation of the laterally attached units into a direction perpendicular to the initial nematic director, eliciting an auxetic response, though, unfortunately, the realization of this interesting approach was not confirmed experimentally. While there are theoretical predictions of a negative Poisson's ratio in smectic- $C$ LCEs [17]; this is yet to be experimentally realized. Additional theoretical work on an anisotropic network of rigid rods, namely, RNA gels, has shown predictions of a negative Poisson's ratio if the connecting chains are non-Gaussian and this behavior is amplified near a mechanical instability [18].

The auxetic response in the nematic LCE that is the subject of this paper occurs at a molecular level, volume is conserved as the system is strained, and there is no evidence of the emergence of porosity for any strain [5]. The auxetic response in the LCE can be described more fully, noting that it occurs in only one axis and beyond a threshold strain (the initial response at low strains is not auxetic). Strictly speaking, this makes the LCE a partial auxetic. The anisotropic nature of LCEs indeed allows for a negative Poisson's ratio in one transverse axis provided that the other transverse axis has a positive Poisson's ratio, thus conserving volume [19]. Interestingly, all of the auxetic LCE materials discovered so far deform under strain via a mechanical Frèedericksz transition (MFT) rather than the more common semisoft elastic (SSE) response. The main features of the two deformation mechanisms seen in 

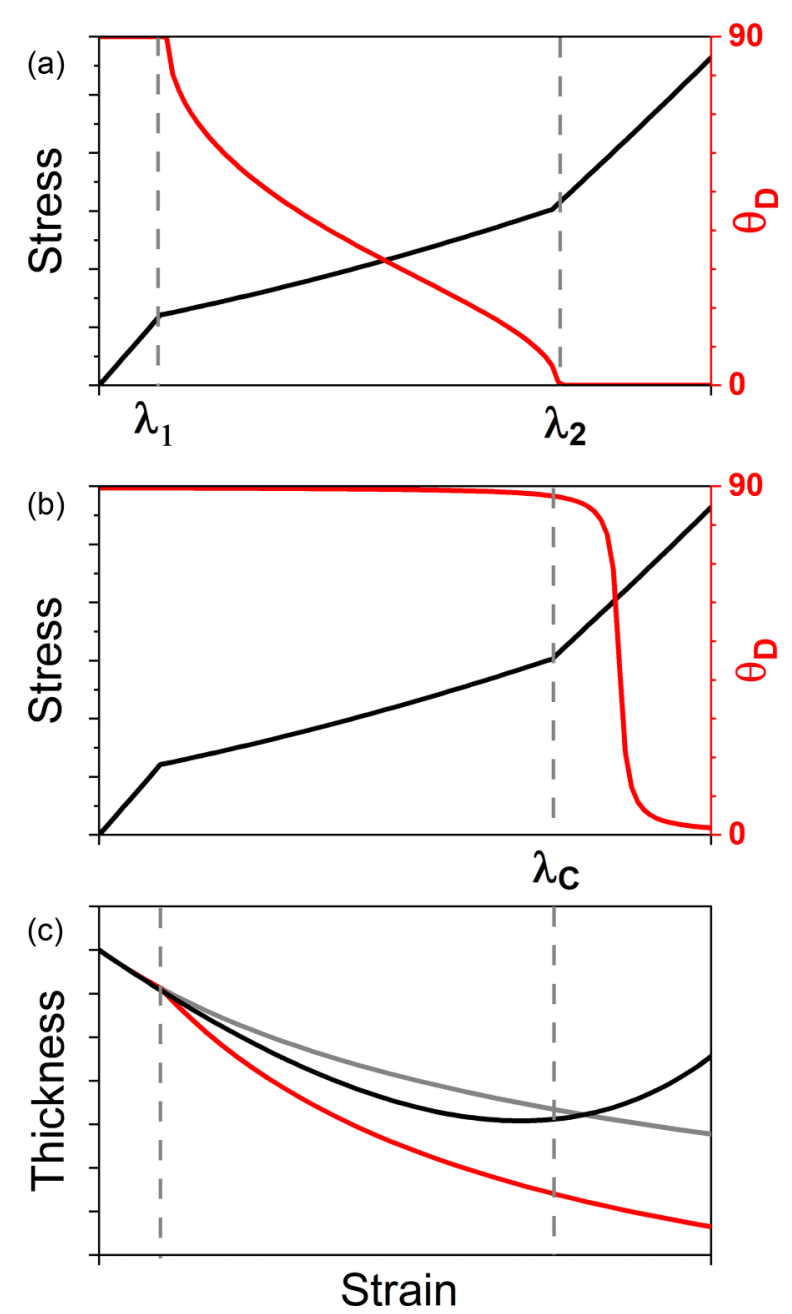

FIG. 1. Illustrative stress-strain responses (black lines) and director behavior (red lines) for (a) the SSE (using relevant SSE theory [31]). (b) The MFT, where the director reorientation is based on theory by Bladon et al. [24] for a sample with a director at $89.8^{\circ}$ to the strain. The critical strain $\lambda_{c}$ is chosen so reorientation begins during the restiffening of the stress response in agreement with experimental findings [26]. (c) Change in thickness for a classical isotropic rubber (grey), a LCE displaying typical SSE behavior (red), and an auxetic LCE (black). The grey and red curves show a reduction in thickness with increasing strain (positive Poisson's ratio), while the auxetic LCE initially has a positive Poisson's ratio that becomes negative (thickness increases with increasing strain) beyond a threshold.

monodomain nematic LCEs can be described as follows. In a SSE response, the liquid crystal director (the average molecular orientation) rotates continuously to align with the applied strain [20-22]. The rotation of the director occurs in counterrotating domains parallel to the stress axis, which minimizes shear affects at the clamped regions, and appears as stripe domains in the sample [21]. During the rotation of the director, the stress-strain response is softened, leading to the term SSE response, see Fig. 1(a). The MFT on the other hand is characterized by a discontinuous director rotation that occurs at a specific critical strain [23-25]. To date, rather few LCEs have been reported to deform via the MFT and all appear to be acrylate based [5,23,26-29]. The stress-strain response associated with the MFT shows that there is again a softening of the response ([26] and Fig. 1(b)). Direct measurements by Mistry et al. on their auxetic LCE suggest that the MFT is implicated in the auxetic response [5,26]. Interestingly, our reanalysis of data by the early pioneers of the MFT response in LCEs suggests they all display a partial auxetic response, identical in nature to that reported by Mistry et al. [23,27-29]. This close link between the MFT and LCE materials that show an auxetic response leads us to suggest that understanding the features that lead to a MFT will underpin our understanding the physics of the auxetic response in LCEs.

The theory that describes the MFT does not predict the stress-strain behavior associated with the transition or a negative Poisson's ratio. However, theoretical work by Bladon et al. shows that an LCE displaying a MFT would experience a decrease in uniaxial order and an increase in biaxial order [24,25]. Indeed, Mitchell et al. reported a MFT in a purely side-chain acrylate-based LCE preceded by a decrease in the macroscopic uniaxial order parameter, $\left\langle P_{200}\right\rangle$, from 0.55 to 0.2 over a strain range from 0 to 0.12 . This agrees with the prediction of a reduction in uniaxial order, though there were no measurements of the biaxial order. After the MFT (at a strain of 0.15$),\left\langle P_{200}\right\rangle$ in their system grew from 0.2 to 0.55 over strains from 0.12 to 0.30 [28]. Mistry et al. also reported a reduction in the sample birefringence with strain prior to the mechanical Frèedericksz transition, followed by a subsequent increase, behavior consistent with a reduction then increase of the $\left\langle P_{200}\right\rangle$ order parameter. The loss of birefringence at the MFT led them to suggest a negative order parameter at that point [26]; see Appendix A for a brief discussion of the negative order in these systems and an illustration of the molecular arrangement for this case. In summary, both theory and experiment suggest that changes in order are important in LCEs that deform via the MFT.

The aim of this paper is to understand the evolution of uniaxial and biaxial order parameters in an LCE displaying a MFT under strain and to hence shed light on the physics that underpins the molecular partial auxetic response seen in this system. We begin by reviewing the relevant LCE theory to bring together as many predictions associated with materials that exhibit a MFT as possible. We then first describe how polarized Raman spectroscopy can be used to deduce the uniaxial order parameters $\left\langle P_{200}\right\rangle$ and $\left\langle P_{400}\right\rangle$ in liquid crystals and apply this for samples strained both parallel and perpendicular to the initial LCE director. This allows us to determine $\left\langle P_{200}\right\rangle$ and $\left\langle P_{400}\right\rangle$ using the well-established uniaxial model which applies to nematic liquid crystals in general. We carefully extend our analysis to explore the potential emergence of biaxiality as predicted by the theory $[24,25,30]$. Based on our findings, we calculate the orientational distribution functions (ODFs) associated with the nematic order and hence offer an explanation for the origin of the auxetic response.

\section{BACKGROUND}

\section{A. Mechanical deformations in nematic LCEs}

We first consider the theoretical description of the MFT which, if our hypothesis of a link between the MFT and the auxetic response is correct, provides important background to 
our evaluation of the auxetic response. As already mentioned, either the SSE or the MFT is observed in a nematic LCE; Fig. 1 shows illustrative tensile load and director reorientation behavior that occurs in the SSE response and the MFT. The SSE response [Fig. 1(a)] can be described as the flattening of the LCE's stress-strain curve beyond a first threshold strain $\left(\lambda_{1}\right)$. After a second threshold strain $\left(\lambda_{2}\right)$, there is a restiffening of the stress-strain response [31]. Nematic rubber elasticity theory predicts a continuous rotation of the nematic director during the softened plateau, a behavior shown extensively in experiments by Küpfer and Finkelmann [20] and others [32,33]. On the other hand, the MFT [Fig. 1(b)] is characterized by a sudden rotation of the nematic director from $90^{\circ}$ to $0^{\circ}$ with respect to the stress axis, after a critical strain $\left(\lambda_{c}\right)$ is reached [26,27]. Figure 1(c) shows the change in thickness that occurs for LCEs deforming via SSE response (red) and the MFT (black). Crucially, while theoretical descriptions for the shapes of the stress-strain curve and the director rotation response exist for semi-soft elasticity [22], only the director rotation response has been accounted for theoretically for the MFT [24]. The stress-strain response, director behavior, and transverse strains of the SSE response were investigated extensively by Verwey et al. [22]. Hence, the illustrative behaviors of the SSE response in Fig. 1 are plotted using the theoretical equations in Ref. [22]. The director behavior of the MFT in Fig. 1 is plotted using theory by Bladon et al. for a sample with a director at $89.8^{\circ}$ to the strain [24]. No theory presently exists for the stress-strain curve of a system undergoing a MFT and the stress strain provided in Fig. 1(b) is an illustration based on previous findings [26]. Likewise, the transverse strains for a system undergoing a MFT is an illustration based on previous findings [5]. Recently, we reported the effect of the initial sample orientation with respect to the strain axis on the the shape of the MFT and the stressstrain curve, showing that the director reorientates much more sharply, and the stress-strain curve flattens, when strained at angles close to $90^{\circ}$ [34].

\section{B. Predicted emergence of biaxiality}

The MFT is the discontinuous rotation of the nematic director with applied strain. During the straining of the sample but before the MFT, one expects, through the coupling between the polymer network and the LC units, a change in the nematic order. Theoretical work by Bladon et al. has shown that the uniaxial order parameter $\left(Q=\frac{1}{2}\left\langle 3 \cos ^{2}(\beta)-1\right\rangle\right)$ reduces and there is an emergence of biaxial ordering $[b=$ $\left.\frac{1}{4}\left\langle\left(1-\cos ^{2}(\beta)\right) \cos (2 \alpha)\right\rangle\right][24,25]$. The angles $\beta$ and $\alpha$ are defined in Fig. 2(a). $Q$ and $b$ are reintroduced as $\left\langle P_{200}\right\rangle$ and $\left\langle P_{220}\right\rangle$, respectively; the latter terminology is commonly used in Raman scattering [35-40]. After the MFT, it is expected that there is a collapse in the biaxial ordering and a return to a uniaxial ordering; however, the macroscopic shape remains biaxial [25].

Finkelmann et al. [30] developed theory describing the elastic anisotropy of strained nematic LCEs when there is no rotation of the nematic director; as is the case for small imposed strains and the MFT. In this work, a relationship was found between the expected changes in the uniaxial order and the biaxial order of an LCE when strained either (a)

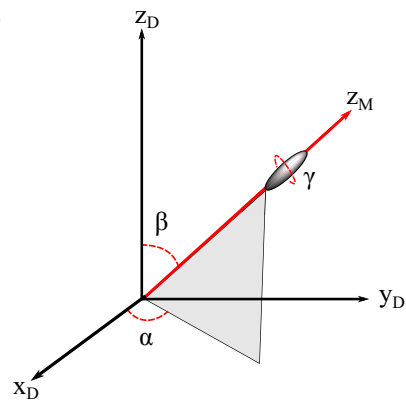

(b)

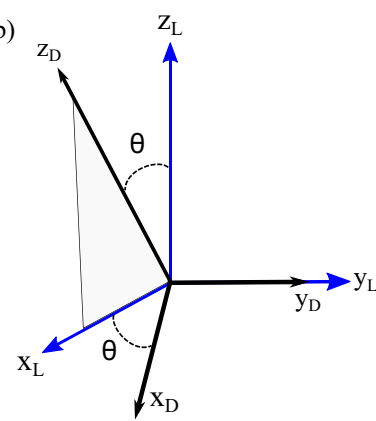

FIG. 2. (a) Schematic showing the molecular frame $\left(Z_{M}\right)$ with respect to the director frame $\left(Z_{D}\right)$ and the relevant Euler angles. (b) Schematic showing the director frame w.r.t the laboratory frame $\left(\mathrm{Z}_{\mathrm{L}}\right)$ and the angle between the laser polarization and sample director $(\theta)$.

parallel or perpendicular to the director. The theoretical work begins with the (shear-free) elastic free-energy of a nematic rubber. Due to mechanical-nematic coupling, changes in the nematic order are accounted for by including the step length tensor in the unstrained state, $\underline{l}^{0}=\operatorname{Diag}\left[l_{\perp}^{0}, l_{\perp}^{0}, l_{\|}^{0}\right]$, and after an imposed strain, $\underline{l}=\operatorname{Diag}\left[l_{x}, \overline{\bar{l}}_{y}, l_{z}\right]$ [30]. The form of elastic free-energy, including changes in orientational order due to imposed strain, is given by the equation

$$
F_{\mathrm{el}}=\frac{1}{2} \mu\left(\lambda_{x x}^{2} \frac{l_{\perp}^{0}}{l_{x}}+\frac{1}{\left(\lambda_{x x} \lambda_{z z}\right)^{2}} \frac{l_{\perp}^{0}}{l_{y}}+\lambda_{z z}^{2} \frac{l_{\|}^{0}}{l_{z}}\right) .
$$

In Eq. (1), $\mu$ is the shear modulus, $\lambda_{i i}$ are extensions in the $i i$ th directions, $l_{i}$ are the the diagonal elements of the step length tensor, $\underline{l}$, for strains applied parallel or perpendicular to the director. $l_{\|}^{0}$ is the unstrained step length parallel to the director, and $l_{\perp}^{0}$ is the unstrained step length perpendicular to the director. The coordinate system can be seen in Fig. 3(a), where the nematic director is in the $z$ direction, and extensions perpendicular to the director are in the $x$ direction while extensions parallel to the director are in the $z$ direction; $y$ is then the sample thickness. It should be noted that Eq. (1) implies constant volume; this is known to be valid for the auxetic LCE used here [5]. The step lengths in the unstrained state $\left(l_{\|}^{0}, l_{\perp}^{0}\right)$ are related to the uniaxial order in the unstrained state, $Q_{0}$. The step lengths after an applied strain $\left(l_{x}, l_{y}, l_{z}\right)$ are related to the new orientational ordering in the system. Hence, the

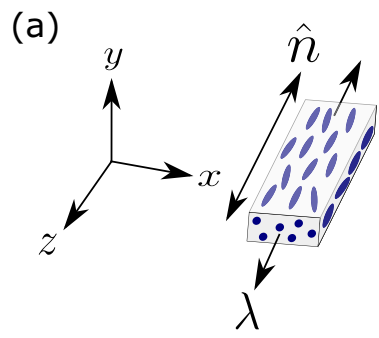

(b)

FIG. 3. (a) Perpendicular geometry refers to the strain being applied perpendicular to the initial nematic director (along $x$ ). (b) Parallel geometry refers to the strain being applied parallel to the initial nematic director (along $z$ ). Thickness is the sample geometry in the $y$ axis. 
step length tensor holds the information on the anisotropy of the network and the order of the liquid crystalline units. The changes in the uniaxial and biaxial order as a function of strain are given by

$$
\begin{gathered}
\delta Q_{\|}=\frac{\mu}{F_{Q}^{\prime \prime}} \frac{3}{\left(1+2 Q_{0}\right)\left(1-Q_{0}\right)} \epsilon, \\
\delta Q_{\perp}=-\frac{\mu}{2 F_{Q}^{\prime \prime}} \frac{3}{\left(1+2 Q_{0}\right)\left(1-Q_{0}\right)} \epsilon, \\
b=\frac{\mu}{2 F_{b}^{\prime \prime}} \frac{3}{1-Q_{0}} \epsilon,
\end{gathered}
$$

where $\delta Q_{\|}$is the change in the uniaxial nematic order when strained parallel to the director, $\delta Q_{\perp}$ is the change in the uniaxial nematic order when strained perpendicular to the director, and $b$ is the emergence of a biaxial order parameter when the sample is strained perpendicular to the director. Here $\epsilon=\lambda-1$ is the strain, $F_{Q}^{\prime \prime}$ is the uniaxial stiffness, and $F_{b}^{\prime \prime}$ is the biaxial stiffness. The uniaxial stiffness and the biaxial stiffness are measurements of how resistant the material is to changes in the uniaxial and biaxial orders, respectively. Finkelmann et al. [30] showed that, by taking a freely jointed chain model, $F_{Q}^{\prime \prime}$ and $F_{b}^{\prime \prime}$ can be related to various material properties through the equation

$$
F_{Q}^{\prime \prime}=F_{b}^{\prime \prime}\left(1+\frac{2}{3}\left(\frac{T^{*}-T}{T_{\mathrm{ni}}-T^{*}}\right) \frac{Q_{\mathrm{ni}}}{Q_{0}(T)}\right),
$$

where $T_{\mathrm{ni}}$ is the the nematic-isotropic transition temperature of the material, $Q_{\mathrm{ni}}$ is the uniaxial order at $T_{\mathrm{ni}}, T$ is the temperature at measurement, $Q_{0}(T)$ is the uniaxial order parameter of the unstrained LCE at $T$, and $T^{*}$ is the critical temperature and is typically close to $T_{\mathrm{ni}}[30,31]$. The $T_{\mathrm{ni}}$ of this sample is very high (extrapolated to be $345 \pm 20^{\circ} \mathrm{C}$ ) and actually above the sample degradation temperature of $330^{\circ} \mathrm{C}[26]$. At room temperature $\left(20^{\circ} \mathrm{C}\right)$ the birefringence of the sample is 0.12 , which we take to be a good indicator of $Q_{0}(T)$. The supercritical nature of the LCEs makes determination of $Q_{\mathrm{ni}}$ difficult [30], however, the closest birefringence measurement to $T_{\mathrm{ni}}$ is 0.01 [26]. The $Q_{\mathrm{ni}} / Q_{0}(T)$ component of Eq. (5) at room temperature $\left(20^{\circ} \mathrm{C}\right)$ will be of the order of 0.08 . We take $T^{*}$ to be $\sim 1{ }^{\circ} \mathrm{C}$ larger than $T_{\mathrm{ni}}$, which is a valid assumption to make [30,31], and set $T$ to $20^{\circ} \mathrm{C}$; the component of Eq. (5) containing $T_{\mathrm{ni}}, T^{*}$, and $T$ will be of the order of 325. Substituting the values of the material, we find $F_{Q}^{\prime \prime} / F_{b}^{\prime \prime}$ of the order of 27, thus we expect the material is far more susceptible to biaxial distortions in order than uniaxial ones as we are well below $T_{\mathrm{ni}}\left(T-T_{\mathrm{ni}}=325^{\circ} \mathrm{C}\right)$. Additionally, because the material exhibits a MFT at a strain of 1.18 [26], the director remains perpendicular to the strain axis up to a large value of strain. Therefore, we expect when this material is strained at room temperature, it will display large biaxial order parameters over a wide strain range as predicted by the prevailing theory $[24,25,30]$.

The theories presented herein consider only the low rank uniaxial and biaxial order parameters $(Q$ and $b)$. However, Raman spectroscopy allows for determination of additional higher rank order parameters $\left(\left\langle P_{400}\right\rangle,\left\langle P_{420}\right\rangle\right.$, and $\left.\left\langle P_{440}\right\rangle\right)$ to get a more complete view of the orientational distribution of molecules in the phase. This is a distinction which will

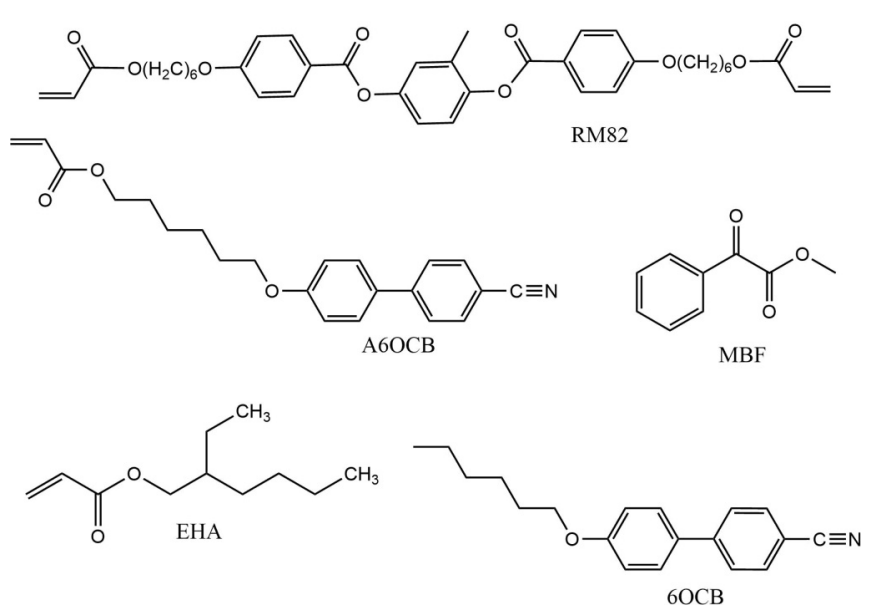

FIG. 4. Structures of the chemicals used in the synthesis of the LCE.

become important in relating biaxial order to the negative Poisson's ratio in these systems.

\section{EXPERIMENTAL SECTION}

\section{A. Materials and synthesis of the LCE}

The LCE was created using the method described by Mistry et al. [26]. The LCE is made from a mixture of the materials shown in Fig. 4. These are the monofunctional reactive mesogen 6-(4-Cyano-biphenyl-4'-yloxy)hexyl acrylate $(\mathrm{A} 6 \mathrm{OCB})$, the bifunctional reactive mesogen 1,4-bis[4-(6-acryloyloxyhex-yloxy)benzoyloxy]-2-methylbenzene (RM82), and nonmesogenic 2-ethylhexyl acrylate (EHA). The nonreactive mesogen $4^{\prime}$-hexyloxybipheny (6OCB) is added to the monomer mixture to broaden its nematic phase, allowing the polymerization to be templated in the nematic phase at room temperature. The 6OCB is washed out after polymerization. Methyl benzoylformate (MBF) is a UV photoinitiator. $\mathrm{A} 6 \mathrm{OCB}, 6 \mathrm{OCB}$, and $\mathrm{RM} 82$ were purchased from Synthon Chemicals $\mathrm{GmbH}$, and EHA and MBF from Sigma Aldrich. The monomer mixture (see Table I for proportions of the components) is nematic at room temperature, with a $T_{\mathrm{ni}}$ at $36.6^{\circ} \mathrm{C}$ [26].

Molds are created using the same methods that are employed to produce highly monodomain low molar mass LC electro-optic devices. 100- $\mu$ m-thick Melinex (DuPont Teijin Films) is used as the top substrate, as this is readily removed following polymerization, while the bottom substrate is con-

TABLE I. Components of the LCE monomer and of the final LCE film.

\begin{tabular}{lcc}
\hline $\begin{array}{l}\text { Chemical } \\
\text { name }\end{array}$ & $\begin{array}{c}\text { Monomer compostion } \\
(\mathrm{mol} \%)\end{array}$ & $\begin{array}{c}\text { De-swollen LCE } \\
\text { composition (mol \%) }\end{array}$ \\
\hline A6OCB & $14.6 \pm 0.1$ & $34.4 \pm 0.2$ \\
6OCB & $55.9 \pm 0.1$ & - \\
RM82 & $7.1 \pm 0.1$ & $16.6 \pm 0.1$ \\
EHA & $20.9 \pm 0.1$ & $49.0 \pm 0.1$ \\
MBF & $1.50 \pm 0.1$ & \\
\hline \hline
\end{tabular}


ventional glass. The substrates are spin coated with a $0.5 \mathrm{wt}$ $\%$ solution of polyvinyl alcohol and deionized water and the substrates are rubbed to create planar alignment. The mold is assembled with the separation of the substrates defining the final film thickness.

To create the LCE, A6OCB, 6OCB, and RM82 are placed in a vial and melted together at $110^{\circ} \mathrm{C}$ for $5 \mathrm{~min}$. EHA and $\mathrm{MBF}$ are added to the mixture and the materials are cooled to $40^{\circ} \mathrm{C}$ with a magnetic stirring bead set at $70 \mathrm{rpm}$ for $4 \mathrm{~min}$. The molds are filled by capillary action at $40^{\circ} \mathrm{C}$ to ensure isotropic filling. The molds are then cooled to room temperature and left for $20 \mathrm{~min}$ to ensure good alignment of the nematic phase. Finally, the LCE is cured to complete polymerization using a UV source. The result is a monodomain nematic LCE swollen with 6OCB. The 6OCB is removed by submerging the swollen LCE in methanol and dichloromethane for $8 \mathrm{~h}$. The deswollen LCE is dried at $40^{\circ} \mathrm{C}$ for $4 \mathrm{~h}$. This process results in highly uniform, monodomain LCE films of dimensions typically $70 \mathrm{~mm} \times$ $20 \mathrm{~mm} \times 0.1 \mathrm{~mm}(\mathrm{~L} \times \mathrm{W} \times \mathrm{T})$ with the director in the plane of the film at any desired angle with respect to the long axis. Herein we use parallel and perpendicular geometries with respect to nematic director and the straining direction and thickness to denote the sample dimension in the $y$ axis. The geometries of the LCE films can be seen in Fig. 3.

\section{B. Raman spectroscopy}

Raman scattering is an inelastic scattering process in which light interacts with the rotational and vibrational modes of a molecule. This results in a shift in the wavelengths of the incident and Raman scattered light. The intensity of the Raman signal is proportional to the square of the differential molecular polarizability. The tensorial nature of the molecular polarizability means that the anisotropic nature of liquid crystal molecules are captured within it and thus liquid crystal order parameters can be determined via Raman spectroscopy $[37,39]$.

The ODF describes the probability of finding a molecule at an angle (or angles) to the director. The complete ODF is a function of the Euler angles $(\alpha, \beta, \gamma)$ shown in Fig. 2. The subscript $\mathrm{M}$ denotes the molecular frame and the $\mathrm{Z}_{\mathrm{M}}$ axis lies along the long axis of the molecule. The subscript $\mathrm{D}$ denotes the director frame and is selected such that the nematic director, $\hat{n}$, is along the $Z_{D}$ axis. For a uniaxial molecule, the ODF is rotationally invariant in $\gamma$. For uniaxial phases, the ODF is rotationally invariant in $\alpha$. Therefore, the ODF for a uniaxial molecule forming a uniaxial phase can be described using only $\beta[37,39]$,

$$
f(\beta)=\sum_{l}^{l=0, \ldots, \infty} \frac{2 l+1}{2}\left\langle P_{l 00}\right\rangle P_{l}(\cos \beta) ; \quad l=\text { even },
$$

where $\left\langle P_{l 00}\right\rangle$ is the uniaxial order parameter of the $l$ th order and $P_{l}$ is the Legendre polynomial of the $l$ th order. Because of the lack of polarity in nematic LCs, the system is indistinguishable under a $180^{\circ}$ rotation about $\hat{n}$ and so only even values of $l$ need to be considered. Raman spectroscopy can determine order parameters up to the fourth rank; hence the relevant Legendre polynomials are given by

$$
\begin{gathered}
P_{2}(\cos (\beta))=\frac{1}{2}\left(3 \cos ^{2}(\beta)-1\right), \\
P_{4}(\cos (\beta))=\frac{1}{8}\left(35 \cos ^{4}(\beta)-30 \cos ^{2}(\beta)+3\right) .
\end{gathered}
$$

It is important to note that since Raman spectroscopy can only determine order parameters to the fourth rank, reconstructed ODFs using only these terms will be inexact.

LCE order parameters are determined by placing a sample with a uniform director in the $x-z$ plane normal to the direction of the incident laser light (incoming from $y$ ). The director and polarization of the laser are in the same plane and measurements are taken. The sample director is then rotated w.r.t to the initial laser polarization $(\theta)$ and subsequent spectra are recorded [37]. Figures 2 and 3 describes the relevant geometry. By selecting an appropriate bond vibration, $\left\langle P_{200}\right\rangle$ and $\left\langle P_{400}\right\rangle$ can be related to the intensity of the corresponding Raman peak [37,39]:

$$
\begin{aligned}
& I_{\|}(\theta) \propto \frac{1}{5}+\frac{4 p}{15}+\frac{8 p^{2}}{15}+\left\langle P_{200}\right\rangle \\
& \times {\left[\frac{1}{21}\left(3+p-4 p^{2}\right)(1+3 \cos (2 \theta))\right] } \\
&+\left\langle P_{400}\right\rangle\left[\frac{1}{280}(1-p)^{2}(9+20 \cos (2 \theta)\right. \\
&\times+35 \cos (4 \theta))], \\
& I_{\perp}(\theta) \propto \frac{1}{15}(1-p)^{2}+\left\langle P_{200}\right\rangle\left[\frac{1}{21}(1-p)^{2}\right]+\left\langle P_{400}\right\rangle \\
& \times {\left[\frac{1}{280}(1-p)^{2}(3-35 \cos (4 \theta))\right] . }
\end{aligned}
$$

To perform the fitting, the depolarization ratio $R(\theta)$ is introduced, which removes the dependence of the fitting on the incident laser intensity, $I_{0}$ :

$$
R(\theta)=\frac{I_{\perp}(\theta)}{I_{\|}(\theta)} .
$$

Generally a fit is made to Eqs. (9) and (10). with data recorded at small increments from $R\left(\theta=0^{\circ}\right)$ to $R\left(\theta=360^{\circ}\right)$ to determine $\left\langle P_{200}\right\rangle,\left\langle P_{400}\right\rangle$, and $p$. However, order parameters can be determined by considering only $R\left(\theta=0^{\circ}\right)$ and $R\left(\theta=90^{\circ}\right)$, provided that the molecular polarizability ratio $(p)$ is known [41]. Raman spectra were recorded as a function of strain using only $R\left(\theta=0^{\circ}\right)$ and $R\left(\theta=90^{\circ}\right)$. To check that $p$ remains constant (within error), the full depolarization ratio at intermediate strain steps of $\sim 0.2$ was determined and fits were made. Doing this significantly speeds up the experimental procedure and reduces the possibility of sample failure under strain.

The Raman depolarization data were collected using a polarized Raman system (Renishaw inVia) comprised of a $532 \mathrm{~nm}, 500 \mathrm{~mW}$ solid state laser (Renishaw) and a Leica polarizing microscope equipped with a rotatable stage. A bespoke rig that allows the elastomer samples to be manually strained is fixed onto the rotatable stage. The LCEs are strained in $0.5 \mathrm{~mm}$ increments and are held for $5 \mathrm{~min}$ 


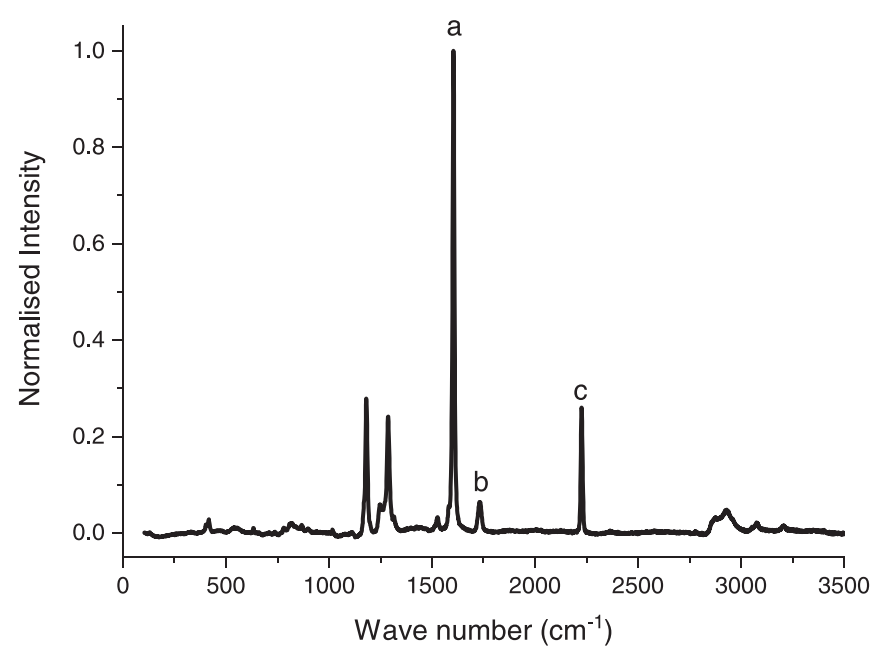

FIG. 5. Raman spectrum of final LCE film showing the symmetric phenyl stretch (a: $1606 \mathrm{~cm}^{-1}$ ), the carbonyl stretch (b: $1730 \mathrm{~cm}^{-1}$ ) and the cyano group stretch $\left(\mathrm{c}: 2250 \mathrm{~cm}^{-1}\right)$.

to allow for stress relaxation before the subsequent Raman spectra are measured. The sample was illuminated via a $20 \times$ lens resulting in a spot size of $\sim 7 \mu \mathrm{m}^{2}$. The laser intensity was chosen to get the best signal to noise ratio for a given strain. Since $p$ can change as a function of intensity [42], $p$ was determined in the unstrained state for a given intensity so fits could be made using the two-point method.

It is important to select an appropriate Raman peak for accurate determination of order parameters [38]. An example of an unpolarized Raman spectrum for the LCE can be seen in Fig. 5. which shows the phenyl stretching mode (a: $1606 \mathrm{~cm}^{-1}$ ), carbonyl stretching mode (b: $1730 \mathrm{~cm}^{-1}$ ) and the cyano stretching mode $\left(\mathrm{c}: 2250 \mathrm{~cm}^{-1}\right)$. We chose the phenyl stretching $\left(1606 \mathrm{~cm}^{-1}\right)$ mode to determine the order parameters as it best satisfies the theoretical assumptions of vibration along the molecular long axis and cylindrical symmetry [38]. The cyano and carbonyl stretching modes $\left(2250 \mathrm{~cm}^{-1}\right.$ and $\left.1730 \mathrm{~cm}^{-1}\right)$ were used to monitor the reorientation of the A6OCB side-chain group and cross-linker RM82, respectively, comparing their behavior to the combined reorientation of the mesogenic groups, all of which exhibit the phenyl stretch. The director angle with respect to the long axis of the sample (see Fig. 3) can be obtained by the full depolarization data via the $\theta$ parameter in Eqs. (9) and (10). The sample is loaded such that the long axis of the material is perpendicular to the incoming laser polarization, $\theta$ is then a fitting parameter and is the angle of the nematic director in the $x-z$ plane. Neither the cyano nor the carbonyl stretching modes were used to determine the order parameters as they return inaccurate values of $\left\langle P_{400}\right\rangle$ [38]. Figure 6 shows the depolarization data of the phenyl stretching mode for the unstrained sample, together with the fit to Eqs. (9) and (10) and the relevant fitting parameters.

\section{Mechanical tests}

Stress-strain measurements were completed in a bespoke rig consisting of two actuators and a load cell enclosed in a temperature-controlled environment. A camera is used to

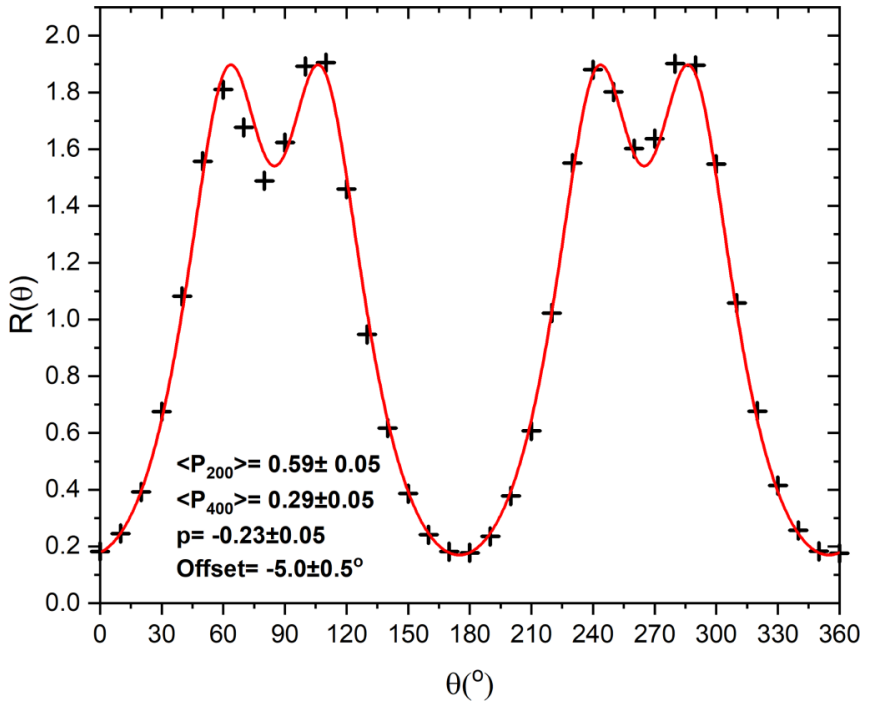

FIG. 6. Depolarization ratio of an unstrained sample determined from the $1606 \mathrm{~cm}^{-1}$ peak. The red line shows the fitting to the data (black crosses) with the values of $\left\langle P_{200}\right\rangle,\left\langle P_{400}\right\rangle, p$, and offset angle, deduced from the fit, also shown.

record images of the LCE to determine local length and width changes, allowing local strains to be deduced. The change in thickness is calculated assuming shear-free and constant volume deformations, known to be satisfied for this LCE. The results and methodology have been reported in full elsewhere [5,26].

\section{RESULTS}

\section{A. Strain parallel to the director $\left(0^{\circ}\right)$}

Raman spectroscopy is used to determine the second$\left(\left\langle P_{200}\right\rangle\right)$ and fourth-rank $\left(\left\langle P_{400}\right\rangle\right)$ uniaxial order parameters for the auxetic LCE strained parallel to its director. In this geometry, the LCE does not display an auxetic response [5]. Figure 7 shows the change in the order parameters as a function of strain with a stress axis at $0^{\circ}$ to the director. We see an increase in $\left\langle P_{200}\right\rangle$ and $\left\langle P_{400}\right\rangle$ from $0.58 \pm 0.05$ and 0.29 \pm 0.05 to $0.69 \pm 0.05$ and $0.39 \pm 0.05$, respectively. The variation in the order parameters follows an approximately linear dependence, in agreement with prevailing theory [30]. The figure also shows the stress-strain response of the LCE over the same range with the typical behavior expected for this geometry.

\section{B. Strain perpendicular to the director $\left(90^{\circ}\right)$}

Figure 8 shows the change in the order parameters as a function of strain, assuming a uniaxial model, for a sample strained perpendicular to its initial director. The figure also shows the director reorientation, with a MFT at a strain of $\sim 1.0$ and the stress-strain curve of the LCE. We see that for strain values between 0 and 0.2 , there is effectively no change in $\left\langle P_{200}\right\rangle$ and $\left\langle P_{400}\right\rangle$; this regime coincides with the first segment of the stress-strain curve (I) which has a relatively large modulus. In this region, there is very little change in the uniaxial order parameters and the orientation of the nematic 

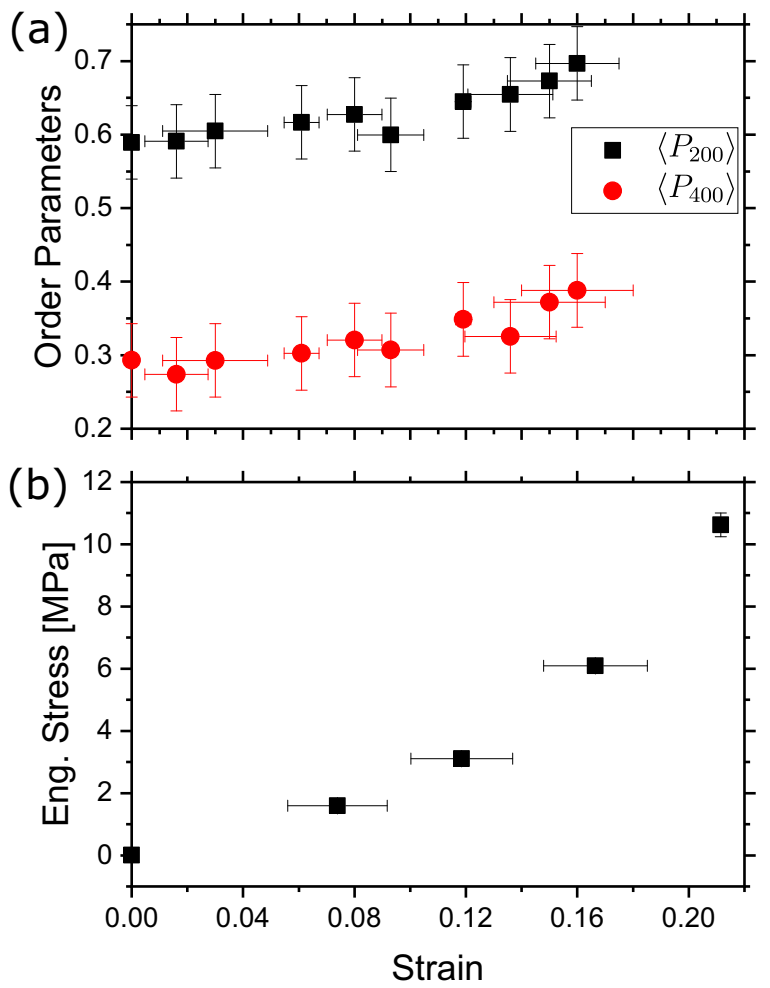

FIG. 7. (a) Order parameters $\left\langle P_{200}\right\rangle$ and $\left\langle P_{400}\right\rangle$ as a function of strain applied parallel to the nematic director. (b) The stress-strain data for the LCE sample.

director remains unchanged. One would expect a relatively large modulus in this region as per predictions by nematic elasticity [31]. After this initial regime, an overall decrease is seen in both $\left\langle P_{200}\right\rangle$ and $\left\langle P_{400}\right\rangle$ as a function of strain until a strain value of $\sim 1.0$. The overall director orientation again remains unchanged in this strain regime [Fig. 8(b)], but a softening of the stress-strain response is seen [Fig. 8(c)]. Interestingly, while $\left\langle P_{200}\right\rangle$ decreases from $0.53 \pm 0.05$ to 0 , $\left\langle P_{400}\right\rangle$ decreases more slowly and appears to plateau, with $\left\langle P_{400}\right\rangle$ eventually crossing over $\left\langle P_{200}\right\rangle$ at a strain of $0.81 .\left\langle P_{400}\right\rangle$ then remains larger than $\left\langle P_{200}\right\rangle$ until a strain of 1.33. A value of $\left\langle P_{400}\right\rangle$ greater than $\left\langle P_{200}\right\rangle$ would suggest a large distribution of molecules $90^{\circ}$ to the overall director, which occurs here in a region well before the reorientation of the director. There is a reemergence of nonzero $\left\langle P_{200}\right\rangle$ values at strains of 1.16; this coincides with the MFT where the director sharply reorients to become parallel to the stress axis. The reorientation of the nematic director coincides with the restiffening of the stressstrain curve. $\left\langle P_{200}\right\rangle$ continues to increase from 0 to a value of $0.17 \pm 0.05$ at a strain of 1.51 .

\section{The behavior of different moieties in the LCE}

Raman scattering is chemically specific so it is possible to investigate whether the two mesogenic units (cross-linker and side chain) are decoupled in any way in their response to strain, analogous to the model suggested in Ref. [15]. Raman data from the $1606 \mathrm{~cm}^{-1}$ line has contributions from both the side chain and the cross-linking unit. However, one can also look at the behavior of the $2250 \mathrm{~cm}^{-1}$ peak,
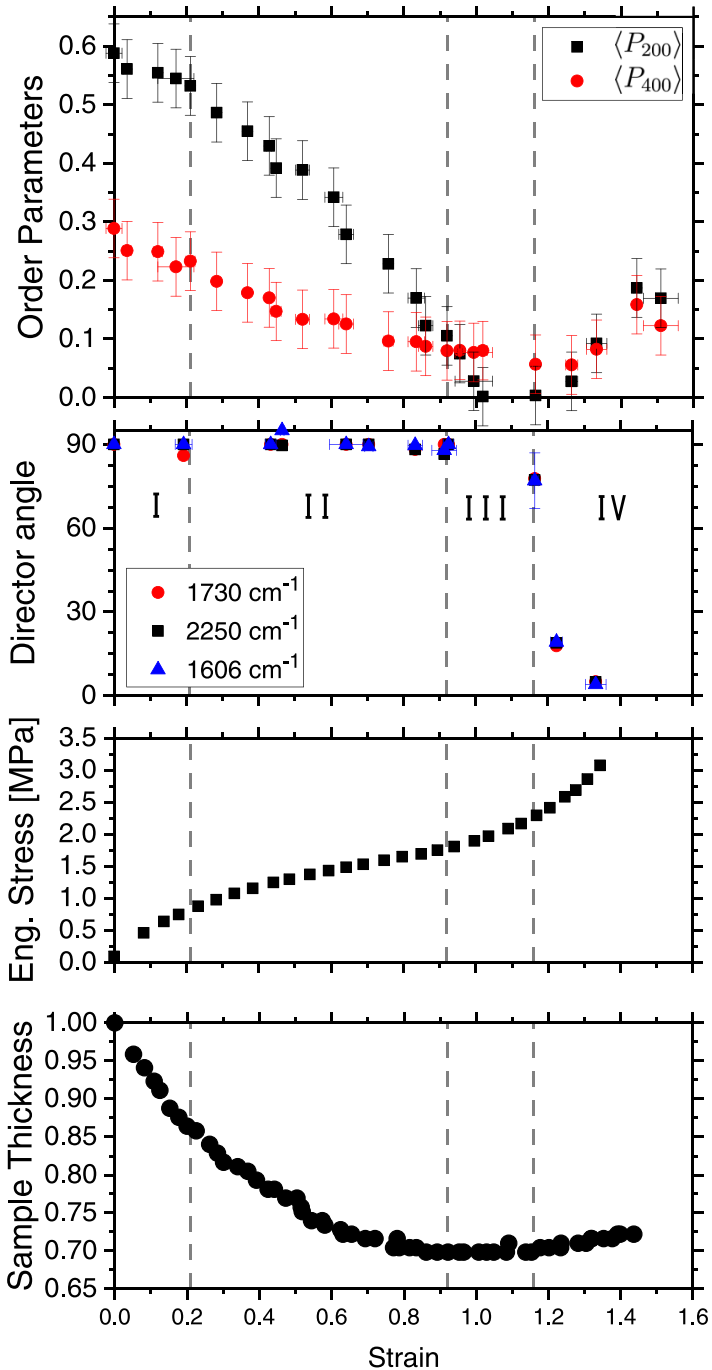

FIG. 8. (a) The uniaxial order parameters $\left\langle P_{200}\right\rangle$ and $\left\langle P_{400}\right\rangle$ as a function of strain for the LCE strained perpendicular to the initial nematic director. (b) Reorientation of the director, determined from fitting to the full depolarization Raman data, showing a MFT at a strain of 1.16. (c) The stress-strain curve of the LCE showing a softening response between the strains of 0.2 and 0.9. (d) Fractional sample thickness of the LCE sample showing the auxetic response emerging at strains near the MFT. The dotted grey lines show points of interest. The first line is the beginning of the softening of the stress response which coincides with the reduction in order clearly demonstrating the coupling of the nematic order and the polymer network. The second line shows the crossing of $\left\langle P_{400}\right\rangle$ and $\left\langle P_{200}\right\rangle$ and the end of the softened stress plateau. Director rotation occurs between the second and final line. The final line shows the reemergence of $\left\langle P_{200}\right\rangle$ order and a sharp rotation of the director. See Mistry et al. $[5,26]$ for more details on stress-strain and thickness measurements.

corresponding to the cyano group, present only in the side chain and the $1730 \mathrm{~cm}^{-1}$ peak, corresponding to the carbonyl group, present only in the cross-linker. We see from Fig. 8(b) that the cross-linking units follow exactly the same reorientation profile as the side-chain units. Therefore, the mesogenic units respond identically to the strain, irrespective 
of whether they are doubly or singly coupled to the acrylate network. The $1730 \mathrm{~cm}^{-1}$ and the $2250 \mathrm{~cm}^{-1}$ vibrations are not used to determine order parameters as they do not satisfy the assumptions required for fitting order parameters via Raman; these are that the vibration is aligned with the long axis of the molecule and that it is a cylindrically symmetric vibration [38].

\section{DISCUSSION}

\section{A. Uniaxial case: Insight into the MFT}

We first note that the order parameter data shown in Figs. 7 and 8 are deduced using the assumption that the sample has uniaxial symmetry. The extent to which our order parameters agree with uniaxial theory is considered in the next subsection. To begin, we consider the insight that the order parameters provide into the nature of the MFT (Fig. 8), noting the following points:

(1) Initially, the mesogenic order is undisturbed and straining pulls on the polymer network, causing an approximately linear stress response. This is region I in Fig. 8.

(2) During the flatter region of the stress response (which is similar in appearance to an SSE response, region II in Fig. 8), the director orientation is unchanged, but there is a marked decrease in both order parameters. The softening of the stress response is due to the increased disorder (and thus degree of freedom) of the mesogenic groups, which allows reorientation of the polymer network. This is a completely different mechanism from that of semisoft elasticity, where the softening of the stress response occurs due to the continuous rotation of the overall nematic director [43]. In our case, during the plateau regime, the order parameter data suggest that an increasing proportion of mesogenic units align with the strain axis, a point discussed further in the next subsection, where we compare the order parameter data to relevant a theoretical model.

(3) Previously, due to the near-zero birefringence of the sample near the MFT, it was deduced that there was zero ordering in the plane of observation and thus the LCE exhibited a negative order parameter [5,26]. See Appendix A for a brief discussion of the negative order in these systems and an illustration of the molecular arrangement for this case. While our measurement of $\left\langle P_{200}\right\rangle=0$ in the same plane is consistent with the previous finding of near zero birefringence, we note the deduced nonzero value of $\left\langle P_{400}\right\rangle$ in this region.

(4) Eventually, the overall director reorients to align with the strain axis, marking the end of the MFT. This occurs during region IV. There is a restiffening of the stress response as the material is pulled parallel to the director with a subsequent increase in order. Interestingly, the continued increase in order with strain in this scenario is quite different (and more marked) than the situation in Fig. 7, potentially explaining the difference in Young's moduli between a sample which has undergone an MFT, being strained parallel to the new director, and one strained parallel to the initial director. In the MFT case, an instantaneous Young's modulus of 5.7 MPa is found whereas an instantaneous Young's modulus of 23.1 MPa is found for a sample pulled parallel to the initial director [26]. One might expect that, due to the different extents in changes of the order parameters, in both these cases the elastic moduli would be different $[30,44]$. We note the highly nonlinear behavior of this system; see Appendix B. Our analysis suggests that the LCE deformation mechanism usually referred to as a MFT is far better described as an order modification transition. This is because the analogy with the electric or magnetic-field cases implied by MFT is unjustified; in the cases of electric and magnetic-field Freedericksz transitions in LCs, there is no change in order parameter, but instead a discontinuous threshold at which the director reorients, with the order staying constant [45]. Our results instead suggest that there is a continuous reduction in order as a function of strain with a subsequent sharp rotation of the director. This is a markedly different response to the continuous rotation of a director with counter-rotating domains that is more typical of the SSE response [21,22,31].

We can compare our order parameter measurements to those determined by Mitchell et al. [23] and Roberts et al. $[27,28]$ who used $\mathrm{X}$-ray scattering to measure $\left\langle P_{200}\right\rangle$ in a side-chain acrylate-based LCE that displayed a MFT. Roberts et al. also found a large decrease in order and both the Mitchell LCE and the LCE studied here remain optically clear under strain [26,28]. The Mitchell LCE was also studied at temperatures deep into the nematic phase, well below $T_{\mathrm{ni}}$, and their discussion suggests that their system deforms through small continuous distributions of the director, rather than through highly localized abrupt changes.

\section{B. Uniaxial case: Deviation from Maier-Saupe theory}

We now discuss how the order parameters, deduced assuming uniaxial symmetry, fit to theoretical predictions. This allows us to investigate whether our initial approach of assuming uniaxial order (which is usual for nematic systems) is justified or whether we need to extend our analysis to determine biaxial order parameters. We compare our $\left\langle P_{200}\right\rangle$ and $\left\langle P_{400}\right\rangle$ data to predictions by Maier-Saupe theory, due to its accuracy in describing conventional low molar mass liquid crystals $[46,47]$.

We see from Fig. 9 that initially (at low strains) the LCE behavior lies near to Maier-Saupe predictions. However, as the strain is increased, $\left\langle P_{200}\right\rangle$ decreases more rapidly than $\left\langle P_{400}\right\rangle$ and the behavior deviates from Maier-Saupe predictions. This trend continues toward the approach of the MFT (black circles). During and after the MFT the deviation from Maier-Saupe predictions continues, in the opposite direction, along the same trend (red circle). It is interesting to note that the measurements where $\left\langle P_{400}\right\rangle>\left\langle P_{200}\right\rangle$ have been predicted by Zannoni and co-workers and observed in fluorophores in membranes [48-51]. This behavior describes an ODF with a large distribution of molecules perpendicular to the overall nematic director. See Appendix $\mathrm{C}$ for selected ODFs constructed using the $\left\langle P_{200}\right\rangle$ and $\left\langle P_{400}\right\rangle$ data.

Figure $8(\mathrm{~b})$ reveals that the cross-linking units follow the same reorientation profile as the side-chain units. This means that the region where $\left\langle P_{400}\right\rangle>\left\langle P_{200}\right\rangle$ cannot be explained by the two types of mesogenic units responding differently to strain. Consequently, we can rule out any model in which the cross-linkers reorient before the side chain that could explain the observation that $\left\langle P_{400}\right\rangle>\left\langle P_{200}\right\rangle$. 


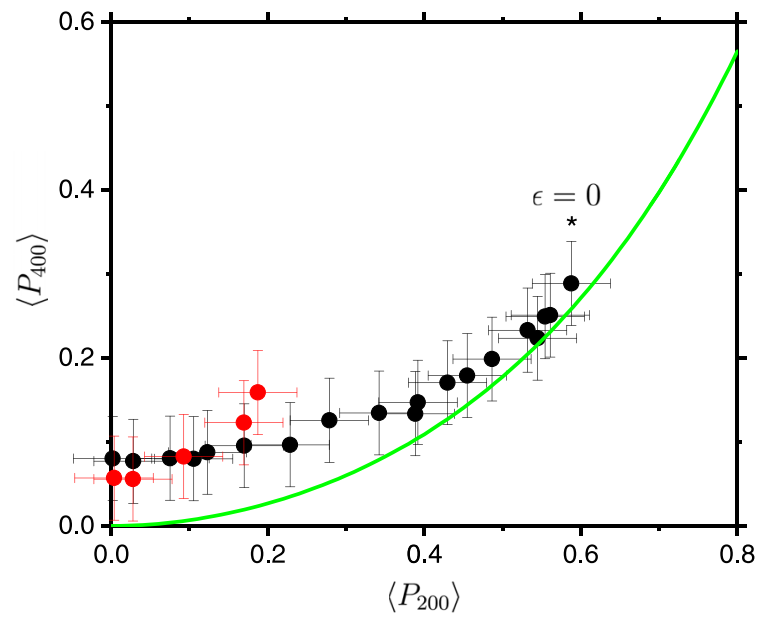

FIG. 9. $\left\langle P_{400}\right\rangle$ plotted as a function of $\left\langle P_{200}\right\rangle$ for the LCE strained perpendicular to the initial director. The black dot highlighted with the asterisk denotes the unstrained state. The black dots denote values of order parameters before the MFT. The red dots denote values of order parameters during and after the MFT. The green line is Maier-Saupe predications of $\left\langle P_{400}\right\rangle$ and $\left\langle P_{200}\right\rangle$ [46].

The key conclusion we can make is that, within error, our data fits with Maier-Saupe theory at low strains. However, there is a systematic deviation from the Maier-Saupe theory at larger strains particularly around the MFT.

\section{The emergence of biaxiality}

Conventional nematic liquid crystals form uniaxial phases and the approach that we have taken so far to deduce the order parameters of our nematic LCE has continued to assume that the system is uniaxial. However, as mentioned previously, theory by Finkelmann et al. [30] describes the case of straining a LCE without rotation of the director, predicting a decrease in the uniaxial order parameters with the emergence of biaxiality. Additionally, it has been reported in low molar mass liquid crystals that a deviation from Maier-Saupe theory can be explained by the emergence of biaxial order [38]. These points, together with the discrepancy in our $\left\langle P_{400}\right\rangle$ data, lead us to consider whether a biaxial model can fit our data. The phase biaxial order parameters are defined as

$$
\begin{gathered}
\left\langle P_{420}\right\rangle=\frac{1}{24}\left\langle\left(7 \cos ^{2}(\beta)-1\right)\left(1-\cos ^{2}(\beta)\right) \cos (2 \alpha)\right\rangle, \\
\left\langle P_{440}\right\rangle=\frac{1}{16}\left\langle\left(1-\cos ^{2}(\beta)\right)^{2} \cos (4 \alpha)\right\rangle, \\
\left\langle P_{220}\right\rangle=\frac{1}{4}\left\langle\left(1-\cos ^{2}(\beta)\right) \cos (2 \alpha)\right\rangle,
\end{gathered}
$$

where $P_{L m 0}$ are a set of the associated Legendre polynomials and are related to Wigner rotation matrix and spherical harmonics via $[36,39,52]$

$$
\begin{aligned}
D_{m 0}^{L}(\alpha, \beta, \gamma) & =\sqrt{\frac{4 \pi}{2 L+1}} Y_{L}^{m *}(\alpha, \beta) \\
& =\sqrt{\frac{(L-m) !}{(L+m) !}} P_{L}^{m}(\cos (\beta)) e^{i m \alpha} .
\end{aligned}
$$

As already noted, $\left\langle P_{220}\right\rangle=b$ is the biaxial order parameter introduced earlier in the LCE theory. Raman scattering can, in principle, be employed to determine the biaxial order parameters in Eqs. (12)-(14) in addition to the uniaxial values, $\left\langle P_{200}\right\rangle$ and $\left\langle P_{400}\right\rangle[36,38]$. However, in practice, it is not possible to directly determine the absolute biaxial contribution to the order parameters from Raman depolarization data, though qualitative biaxial behavior can be deduced by careful analysis of the data [35]. This approach has been used to successfully observe the emergence of biaxial order in a biaxial SmA system [35]; we use the same approach here. We first assume that the molecular biaxial order parameters $\left(\left\langle P_{402}\right\rangle,\left\langle P_{404}\right\rangle,\left\langle P_{202}\right\rangle\right)$ are equal to 0 . Then, the ODF is still rotationally invariant to $\gamma$ but now has dependencies on $\theta$ and $\alpha$. A modification can then be made to Eqs. (9) and (10) to account for phase biaxiality, including contributions from Eqs. (12)-(14). The full equations are given in Appendix D.

To deduce values of the biaxial order parameters we first construct idealized depolarization data from the experimental values of $\left\langle P_{200}\right\rangle$ and $\left\langle P_{400}\right\rangle$ using the experimentally determined value of $p$ in the unstrained state $(p=-0.23)$. This smooths the data and allows us to extrapolate between experimental values. We then make the assumption that the anomalously large values of $\left\langle P_{400}\right\rangle$ that cause our data to deviate from Maier-Saupe behavior (Fig. 9) can be attributed to biaxiality. This is reasonable as this is predicted by theory $[24,25,30]$ and we know that the birefringence of the sample becomes zero at the MFT [26]. We can calculate new $\left\langle P_{400}\right\rangle$ values from $\left\langle P_{200}\right\rangle$ by assuming that the uniaxial contributions, $\left\langle P_{200}\right\rangle$ and $\left\langle P_{400}\right\rangle$, follow Maier-Saupe theory predictions as seen in Fig. 10(a). These values are then fixed and a new fit is made to the reconstructed depolarization data using the full expression, including biaxial terms (see Appendix D), thus obtaining values for $\left\langle P_{420}\right\rangle,\left\langle P_{440}\right\rangle$, and $\left\langle P_{220}\right\rangle$ which can be seen in Fig. 10(b). The qualitative behavior of the biaxial order parameters determined using this approach is known to be valid, but their absolute values are not [35]. An important check of the robustness of this approach is that the values of $\left|\left\langle P_{420}\right\rangle\right|,\left|\left\langle P_{440}\right\rangle\right|$, and $\left|\left\langle P_{220}\right\rangle\right|$ are all within physically meaningful limits $(0.0563,0.0625$, and 0.25 respectively).

It is interesting to now consider the emergence of biaxiality in the LCE under strain (Fig. 10). Our analysis returns very small values for the biaxial order parameters in the linear elastic region (strains $<0.2$ ), where it was already apparent that $\left\langle P_{200}\right\rangle$ and $\left\langle P_{400}\right\rangle$ followed Maier-Saupe behavior. However, we can see that with increasing strain (and decreasing $\left\langle P_{200}\right\rangle$ and $\left\langle P_{400}\right\rangle$ values), there is a clear increase in the magnitude of the biaxiality of the phase, as predicted by theory [30]. The absolute values of all the biaxial order parameters increase until the MFT (strain $\sim 1.0$ ), following which they decrease. Interestingly, and relevantly, the value of $\left\langle P_{220}\right\rangle=b$ becomes increasingly large and negative. The biaxial behavior is consistent with theoretical predictions, where the biaxial order emerges prior to the MFT [30]. Bladon et al. also predicted that after the MFT there is a collapse of the biaxial order and reemergence of uniaxial order [25] $\left\langle P_{220}\right\rangle$ and the other biaxial order parameters show a decrease in magnitude after the MFT, coinciding with the reemergence of uniaxial ordering, in apparent agreement with predictions for the reorientation event [25]. However, while there is a reduction in the biaxial order 



(c)



FIG. 10. (a) Uniaxial order parameters $\left(\left\langle P_{200}\right\rangle\right.$ : black squares, $\left\langle P_{400}\right\rangle$ : red circles) used in the fitting of the biaxial order parameters. The values for $\left\langle P_{400}\right\rangle$ are obtained using Maier-Saupe predictions. (b) Biaxial order parameters $\left(\left\langle P_{420}\right\rangle\right.$ : purple diamonds, $\left\langle P_{440}\right\rangle$ : blue downward triangles $\left\langle P_{220}\right\rangle$ : green upward triangles) as a function of strain. (c) Instantaneous Poisson's ratio of the auxetic LCE as a function of strain which becomes negative at a strain of $\sim 1.00$. See Mistry et al. [5,26] for more details on stress-strain and thickness measurements.

parameters after reorientation of the director, the biaxial order parameters do not collapse to zero as predicted theoretically. While theoretical approaches typically assume that the strains imposed are small and that the polymer chains are Gaussian $[24,25,30]$, this has been shown to not necessarily be the case [53] and is certainly not the case here with strains $>100 \%$. Hence, even after the MFT, the system remains biaxial, which may have an implication in the mechanical properties of the material [54].

\section{Relating biaxiality to the auxetic behavior}

The theory predicting biaxiality in LCEs considers only the $b=\left\langle P_{220}\right\rangle$ order parameter; however, Raman spectroscopy

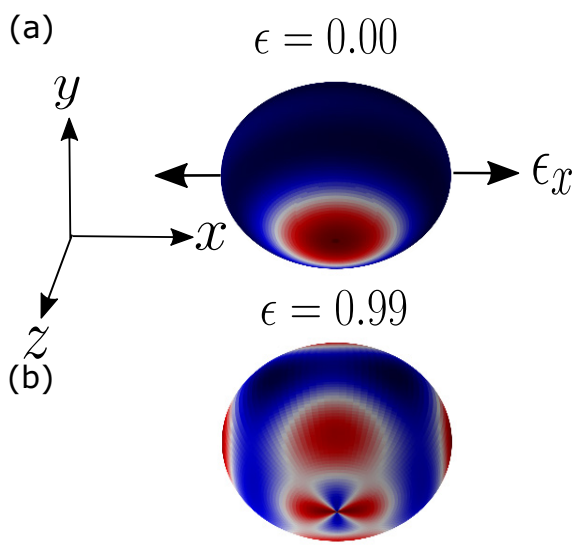

FIG. 11. Truncated ODF plot showing distribution of molecules in the unstrained state (a) and at the beginning of the auxetic response (b). The initial director orientation is along $x$ and the strain axis is in the $y$ direction. The macroscopic sample's thickness occurs is in the $y$ dimension. The red regions correspond to the highest density of molecules and black/blue the lowest.

can determine $\left\langle P_{220}\right\rangle,\left\langle P_{420}\right\rangle$, and $\left\langle P_{440}\right\rangle$. The ability for Raman spectroscopy to probe higher order biaxial terms allows us to propose a mechanism for the auxetic response in this LCE, which occurs above a threshold strain of $\sim 1.0$. Figure 10 shows that the biaxial order parameters emerge rather slowly until a strain of $\sim 0.6$ when $\left\langle P_{220}\right\rangle$ rapidly decreases toward -0.025 at a strain $\sim 1.0$. $\left\langle P_{420}\right\rangle$ changes little during the full strain range. However, $\left\langle P_{440}\right\rangle$ remains relatively constant until a strain of $\sim 0.8$ were it becomes increasingly positive. The increase in $\left\langle P_{440}\right\rangle$ occurs briefly before the onset of a negative Poisson's ratio [5]. Interestingly, the magnitude of $\left\langle P_{220}\right\rangle$ and $\left\langle P_{440}\right\rangle$ are maximal at the point where the Poisson's ratio becomes negative. We believe that the increase of $\left\langle P_{440}\right\rangle$ may provide a possible explanation for the emergence of molecular auxeticity, as follows.

The distribution of molecules (ODF) can be calculated from the order parameters $\left\langle P_{200}\right\rangle,\left\langle P_{400}\right\rangle,\left\langle P_{420}\right\rangle,\left\langle P_{440}\right\rangle$, and $\left\langle P_{220}\right\rangle$. It should be noted that since Raman spectroscopy can only determine order parameters up to the fourth rank, that reconstructed ODFs will be inexact but nonetheless give insight into the molecular distribution of the system [49] (see Supplemental Material [55]). Figure 11 shows the distribution of molecules for strain values of 0 and $\sim 0.99$; the larger strain is at the beginning of the auxetic response.

Figure 11 can be understood as follows. The sample has an initial director oriented along the $z$ axis and is strained along the $x$ axis. In the unstrained state, the molecular distribution is uniaxial with the highest probability found for molecules aligning along the $z$ direction (red region). However, in the auxetic regime, there is now a large distribution of molecular orientations in the $x$ direction, which is along the strain axis. This is a consequence of the contributions of the $\left\langle P_{220}\right\rangle$ order parameter and agrees with prevailing theory [30]. We also see a growing distribution of molecules towards the $y$ axis in the $y z$ plane, presumably a consequence of the combination of $\left\langle P_{420}\right\rangle$ and $\left\langle P_{440}\right\rangle$. The redistribution of molecules toward the $y$ axis on a macroscopic scale would increase the dimensions in that direction, providing an explanation for the emergence of a 
negative Poisson's ratio in this sample in the $y$ direction. Such out-of-plane deformations have been suggested to achieve a molecular auxetic response in liquid crystalline polymers, however, this was not realized experimentally $[15,16]$. Early work on microporous foams has also suggested a similar mechanism, albeit with the formation of voids [56,57], unlike the LCE used here which conserves volume and remains voidless [5].

Here we emphasize that only the relative values of the biaxial order parameters have been deduced, and while their qualitative dependence on strain is robust, we cannot determine the absolute values of these parameters. That means that while our data shows how the emergence of biaxiality can, in principle, result in an auxetic response perpendicular to both the initial director and the strain axis, it cannot quantify the extent of the negative Poisson's ratio.

\section{CONCLUSION}

Our motivation for this paper was twofold. First, we aimed to understand the mechanism of the auxetic response in the material. Second, as a link was implied experimentally between the MFT and the auxetic response, we wished to understand the apparent contradiction in the mechanical behavior of a LCE that underwent a MFT; in particular, why the stress-strain curve was reminiscent of a SSE response.

We determined the evolution of the order parameters of the LCE and related these to the mechanical behavior of the material under strain. The nematic order parameter $\left\langle P_{200}\right\rangle$ clearly decreases to zero at the point of the MFT, which is consistent with previous measurements via $\mathrm{x}$-ray spectroscopy [23,27] and with the determination of zero birefringence which lead to the assumption of a negative order parameter [5]. It is clear from this work that the order changes continuously in the system and that the elastic softening of the material coincides with the reduction in order. The deformation mechanism is not analogous with a Fréedericksz transition, and we suggest that it is more appropriately referred to as an order modification transition in the future rather than a MFT (MFT).

We demonstrated that fitting the Raman data acquired for this system assuming uniaxial symmetry reveals inconsistencies in the relationship between $\left\langle P_{200}\right\rangle$ and $\left\langle P_{400}\right\rangle$-they do not follow Maier-Saupe theory. However, by constraining them to do so, we showed that stretching the LCE induces biaxial order. The emergence of a negative $\left\langle P_{220}\right\rangle$ describes a distribution of molecules around the azimuthal angle and in line with the direction of strain, which is an intuitive result. However, we also see the emergence of a significant population of molecules perpendicular to the strain, in the direction of the observed auxetic response. Excitingly, this occurs at the onset of the observed auxetic threshold and provides a tangible explanation for this response. It is important to note that the theory predicting biaxiality in these systems considers only the $b=\left\langle P_{220}\right\rangle$ term [30] whereas we propose that the higher rank biaxial terms $\left(\left\langle P_{420}\right\rangle\right.$ and $\left.\left\langle P_{440}\right\rangle\right)$ are the drivers of the auxetic response. We hope that this may inspire researchers to consider the higher rank terms in future work.

Questions arise as to whether the uniaxial order parameters become negative [5], a state which is not possible to probe using Raman spectroscopy in this geometry as it is required that the nematic director and the laser polarization are in the same plane. Certainly, $\left\langle P_{200}\right\rangle=0$ in the plane investigated can be indicative of a negative order state, however, we note the nonzero biaxial order parameters in this region. In any case, the potential negatively ordered state occurs in a very small strain range $[5,26]$ and there is clearly rich order parameter behavior occurring before and after the transition, due to imposed strains. LCEs have been developed which have a stable negative ordering [58] in the unstrained state and further experiments will be required to understand whether a negative order parameter that then evolves with strain also results in a negative Poisson's ratio.

In conclusion, we suggest that materials that exhibit a MFT must also develop significant biaxiality, as predicted theoretically $[24,25,30]$. Doing so allows an auxetic response in the LCE, making a MFT a signature of auxetic behavior. It is noteworthy that the material studied here, in which the auxetic response was first measured, satisfies the condition in Eq. (5) for biaxiality to dominate as $T_{\mathrm{ni}}>330^{\circ} \mathrm{C}$ [26] and measurements are taken at $T=20^{\circ} \mathrm{C}$, therefore we expect the biaxial stiffness of the material to be much lower than the uniaxial stiffness. We can further suggest that an auxetic response in LCEs is more likely to occur around room temperature in LCEs with a high $T_{\mathrm{ni}}$ and it will be seen above the glass transition temperature where the system can behave elastically. This describes the acrylate systems that have been reported to exhibit a MFT, at least one of which [28] appears to undergo an auxetic response. Questions remain about whether the magnitude and threshold of the auxetic response can be predicted but the first important steps in determining what elicits an auxetic response in LCEs have now been taken.

Data sets associated with this work will be made available at Ref. [59].

\section{ACKNOWLEDGMENTS}

The authors acknowledge useful discussions with colleagues, especially Martin Čopič, Luka Cmok, and Andrej Petelin of the Jožef Stefan Institute, Mojca Čepič of the University of Ljublana and Peter Hine of the University of Leeds. T.R. thanks the University of Leeds Alumni Fund. D.M. thanks the English-Speaking Union for funding an Lindemann Fellowship. Z.Z., J.M., and H.F.G. thank the EPSRC for support (EP/M009521/1).

\section{APPENDIX A: ON THE NEGATIVE ORDER PARAMETER STATE OF THE LCES}

The LCE studied in the main body of the paper was previously studied under cross polarizers to evaluate birefringence qualitatively as a function of strain $[5,26]$. It was shown that there was near-zero birefringence close to the strain associated with the MFT. A state of negative order was deduced from the observation of near-zero birefringence. A schematic of the molecular arrangement of a negative order parameter can be seen in Fig. 12. 


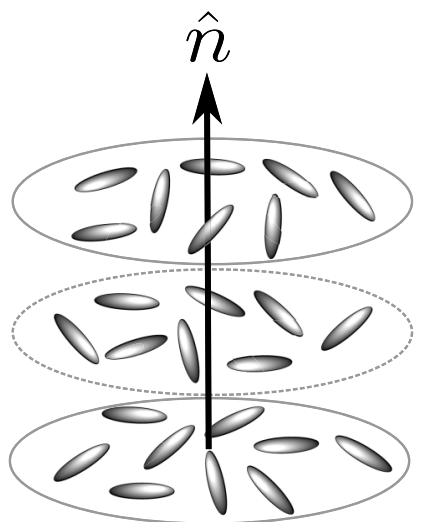

FIG. 12. Schematic representation of a nematic phase with a negative order parameter. The molecules are aligned perpendicular to the nematic director and are perfectly disordered. The resultant phase would produce no birefringence colours under a polarized optical microscope when viewed along the director. When probed with Raman spectroscopy, with the incident laser parallel to the nematic director, the measured uniaxial order parameter, $\left\langle P_{200}\right\rangle$, would also be zero.

\section{APPENDIX B: ELASTIC MODULI AS A FUNCTION OF STRAIN}

It is noted in the main body of paper that the stress-strain behavior of the LCE is, in fact, nonlinear. We show the elastic moduli as a function of strain for the sample strained perpendicular to the initial director direction in Fig. 13. In the main body of the paper, we suggest a connection between the stress-strain behavior of an LCE and the change in order parameters as a function of strain. For the initially perpendicular sample, we quote an instantaneous Young's modulus of around $5 \mathrm{MPa}$ for strains up to 0.2 . In the region where the order parameters decrease rapidly (strains from 0.2 to 0.9 ), the modulus is small and approximately constant (the system is softened). At the MFT, when the order parameters start to increase again (strains greater than $\sim 1.1$ ), the modulus is initially $\sim 5.7 \mathrm{MPa}$ and rises rapidly. In contrast, for the sample that is strained parallel to the initial director direction, an instantaneous Young's modulus of $23.1 \mathrm{MPa}$ is observed at very low strains [26]. This much larger modulus can be explained by noting that the changes in order parameter are very small. Conversely, large changes in order correspond to a softening (small modulus), as seen in Fig. 13.

\section{APPENDIX C: SELECTED ORIENTATION DISTRIBUTION FUNCTIONS ASSUMING UNIAXIAL SYMMETRY}

In the main body of the paper, uniaxial order parameters $\left(\left\langle P_{200}\right\rangle\right)$ and $\left.\left\langle P_{400}\right\rangle\right)$ are deduced from Raman spectroscopy assuming a uniaxial symmetry in the phase. The resulting order parameters are compared to predictions from MaierSaupe theory and a clear deviation from theory is observed for intermediate and large strains. As mentioned, in the main body of the paper, the ODF for the molecules can be constructed, but where only $\left\langle P_{200}\right\rangle$ and $\left\langle P_{400}\right\rangle$ are used, it will be inexact as other terms are strictly needed. However, an information theory approach can be used to deduce the ODF,
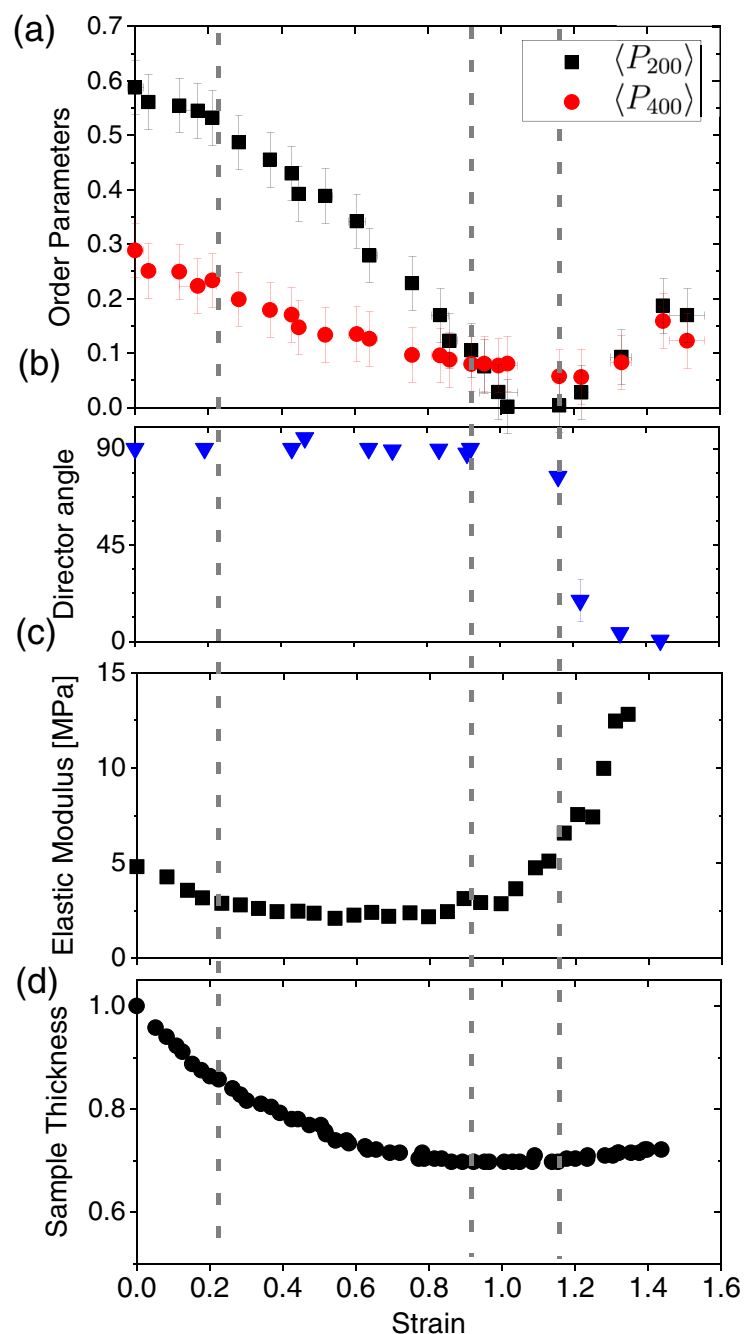

FIG. 13. (a) Uniaxial order parameters as a function of strain. (b) Director behavior determined from the $1606 \mathrm{~cm}^{-1}$ peak showing reorientation of the director; which is indicative of the MFT. (c) Elastic moduli of the LCE as function of strain. (d) Sample thickness as a function of strain. See Fig. 8. for full description of the different regions.

as described briefly below (we point the reader to a book chapter by Zanonni [49] for a more in-depth discussion with respect to liquid crystal phases).

In the information theory approach, problems with truncations are accounted for by finding the distribution which maximizes the informational entropy of the distribution. This leads to an exponential form of the ODF [49]:

$$
f(\beta)=\exp \left\{\sum_{l=0}^{l} a_{l} P_{l}(\cos (\beta))\right\} .
$$

Thus, knowing order parameters up to fourth rank results in a distribution function of the form [49]

$$
f(\beta)=\frac{\exp \left(a_{2} P_{2}(\cos \beta)+a_{4} P_{4}(\cos \beta)\right)}{\int_{0}^{\pi} d \beta \sin \beta \exp \left(a_{2} P_{2}(\cos \beta)+a_{4} P_{4}(\cos \beta)\right)},
$$

where $a_{2}$ and $a_{4}$ are constants to be from the determined from known values of $\left\langle P_{200}\right\rangle$ and $\left\langle P_{400}\right\rangle$ by solving a system of 
linear equations [49]:

$$
\begin{aligned}
\left\langle P_{2}\right\rangle & =\frac{\int_{0}^{\pi} P_{2}(\cos \beta) \exp \left[b_{2} P_{2}(\cos \beta)+b_{4} P_{4}(\cos \beta)\right] \sin \beta d \beta}{\int_{0}^{\pi} \exp \left[b_{2} P_{2}(\cos \beta)+b_{4} P_{4}(\cos \beta)\right] \sin \beta d \beta}, \\
\left\langle P_{4}\right\rangle & =\frac{\int_{0}^{\pi} P_{4}(\cos \beta) \exp \left[b_{2} P_{2}(\cos \beta)+b_{4} P_{4}(\cos \beta)\right] \sin \beta d \beta}{\int_{0}^{\pi} \exp \left[b_{2} P_{2}(\cos \beta)+b_{4} P_{4}(\cos \beta)\right] \sin \beta d \beta} .
\end{aligned}
$$

The values of $a_{2}$ and $a_{4}$ thus determined can then be inserted into Eq. (C2) and the ODF can be constructed. The results of this are seen in Fig. 14. When assuming a uniaxial model, the deduced order parameters reveal an increasing population of molecules in the direction toward the strain axis. This is particularly apparent in the regions where $\left.\left\langle P_{400}\right\rangle\right\rangle\left\langle P_{200}\right\rangle$ (uniaxial model) and has been studied extensively by Zannoni et al. [49-51]. In Fig. 14, the ODFs are shown for selected strains, clearly showing the increased population of molecules perpendicular to the initial director and in line with the strain axis (blue lines at $\beta=90^{\circ}$ ).

\section{APPENDIX D: RAMAN SPECTROSCOPY TO DETERMINE BIAXIAL ORDERING}

In principle, Raman spectroscopy can be used to determine the phase biaxial order parameters $\left(\left\langle P_{420}\right\rangle,\left\langle P_{440}\right\rangle\right.$, and

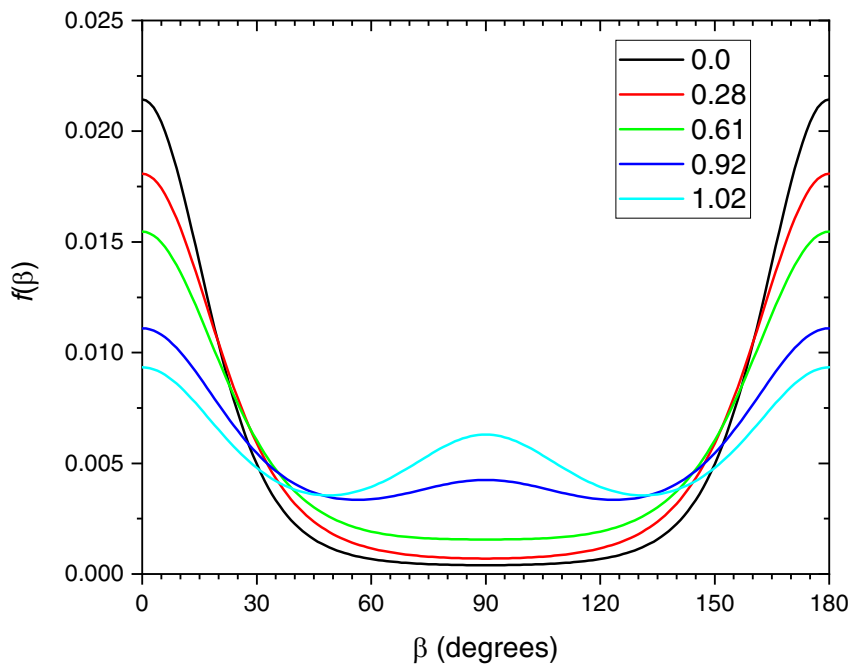

FIG. 14. ODFs for the perpendicularly strained LCE constructed from the order parameter data deduced with the assumption of a uniaxial distribution of molecules [Fig. 8(a)]. The ODFs are constructed using information theory. Black line: 0.00 strain. Gaussian distribution of molecules singly peaked at $\beta=0^{\circ}$; at this strain, our order parameter data agrees with Maier-Saupe theory. Red line: 0.28 strain. Reduction in $\left\langle P_{200}\right\rangle$ and $\left\langle P_{400}\right\rangle$ broadens the distribution, however, the distribution is still Gaussian and singly peaked at $\beta=0^{\circ}$; here our order parameters still closely match predictions by Maier-Saupe theory. Green line: 0.61 strain. Further reduction in $\left\langle P_{200}\right\rangle$ and $\left\langle P_{400}\right\rangle$ broadens the distribution and there is a deviation from Maier-Saupe theory. Dark blue line: 0.92 strain. $\left\langle P_{200}\right\rangle$ reduces faster than $\left\langle P_{400}\right\rangle$ the distribution now falls out of the Gaussian regime and becomes doubly peaked at $\beta=0^{\circ}$ and $\beta=90^{\circ}$. Cyan line: 1.02 strain. There is a large distribution of molecules at $\beta=90^{\circ}$. This ODF corresponds to the beginning of the MFT. $\left.\left\langle P_{220}\right\rangle\right)$ by modifying the intensity equations of the Raman spectra [Eqs. (9) and (10)]. This approach has been described in detail in Zhang et al. [35] and the main assumptions are included in the Discussion section of the main body of the paper. Equations (D1) and (D2) are the modified Raman intensity equations which include the phase biaxiality terms:

$$
\begin{aligned}
I_{\|}= & \frac{2}{15}\left(5\left(1+2 p+3 p^{2}\right)+(p-1)^{2} \cos 2 \Omega\right) \\
& -\frac{1}{42}\left\langle P_{200}\right\rangle(p-1)(5+9 p-(p-1) \cos 2 \Omega \\
& +3(3+4 p) \cos 2 \chi+3(3+4 p) \cos 2(\chi+\Omega)) \\
& \times(1+3 \cos 2 \theta)+\frac{1}{17920}\left\langle P_{400}\right\rangle(p-1)^{2} \\
& \times(6 \cos 2 \Omega+5(6+8 \cos 2 \chi+7 \cos 4 \chi \\
& +8 \cos 2(\chi+\Omega)+7 \cos 4(\chi+\Omega) \\
& +14 \cos 2(2 \chi+\Omega)))(9+20 \cos 2 \theta+35 \cos 4 \theta) \\
& -\frac{2}{7}\left\langle P_{220}\right\rangle(p-1)(5+9 p-(p-1) \cos 2 \Omega \\
& +3(3+4 p) \cos 2 \chi+3(3+4 p) \cos 2(\chi+\Omega)) \\
& \times \sin ^{2} \theta+\frac{3}{224}\left\langle P_{420}\right\rangle(p-1)^{2}(6 \cos 2 \Omega \\
& +5(6+8 \cos 2 \chi+7 \cos 4 \chi+8 \cos 2(\chi+\Omega) \\
& +7 \cos 4(\chi+\Omega)+14 \cos 2(2 \chi+\Omega))) \\
& \times(5+7 \cos 2 \theta) \sin ^{2} \theta+\frac{1}{32}\left\langle P_{440}\right\rangle(r-1)^{2} \\
& \times(6 \cos 2 \Omega+5(6+8 \cos 2 \chi+7 \cos 4 \chi \\
& +8 \cos 2(\chi+\Omega)+7 \cos 4(\chi+\Omega) \\
& +14 \cos 2(2 \chi+\Omega))) \sin ^{4} \theta,
\end{aligned}
$$

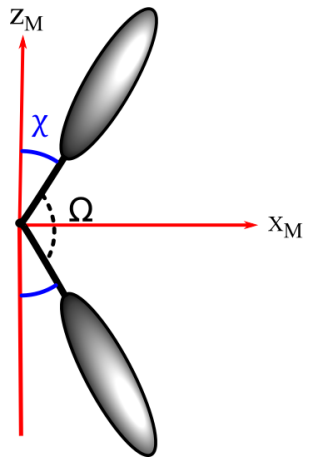

FIG. 15. Schematic of the fitting model for the deduction of phase biaxiality via Raman spectroscopy first introduced for bentcore molecules. The schematic shows the tilt angle with respect to the molecular long axis $(\chi)$ and the bend angle $(\Omega)$ of a bent-core molecule, which is $180^{\circ}$ for the LCEs. The experimental geometry is exactly the same as in Fig. 2 in the main body of the paper. 


$$
\begin{aligned}
I_{\perp}= & \frac{1}{30}(p-1)^{2}(5+3 \cos 2 \Omega)+\frac{1}{84}\left\langle P_{200}\right\rangle \\
& \times(p-1)^{2}(1+3 \cos 2 \Omega+6 \cos 2 \chi \\
& +6 \cos 2(\chi+\Omega))-\frac{1}{17920}\left\langle P_{400}\right\rangle(p-1)^{2} \\
& \times(6 \cos 2 \Omega+5(6+8 \cos 2 \chi+7 \cos 4 \chi \\
& +8 \cos 2(\chi+\Omega)+7 \cos 4(\chi+\Omega) \\
& +14 \cos 2(2 \chi+\Omega)))(-3+35 \cos 4 \theta) \\
& +\frac{1}{14}\left\langle P_{220}\right\rangle(p-1)^{2}(1+3 \cos 2 \Omega+6 \cos 2 \chi \\
& +6 \cos 2(\chi+\Omega))+\frac{3}{896}\left\langle P_{420}\right\rangle(p-1)^{2} \\
& \times(6 \cos 2 \Omega+5(6+8 \cos 2 \chi+7 \cos 4 \chi \\
& +8 \cos 2(\chi+\Omega)+7 \cos 4(\chi+\Omega)
\end{aligned}
$$

$$
\begin{aligned}
& +14 \cos 2(2 \chi+\Omega)))(1+7 \cos 4 \theta)+\frac{1}{128} \\
& \times\left\langle P_{440}\right\rangle(p-1)^{2}(6 \cos 2 \Omega+5(6+8 \cos 2 \chi \\
& +7 \cos 4 \chi+8 \cos 2(\chi+\Omega)+7 \cos 4(\chi+\Omega) \\
& +14 \cos 2(2 \chi+\Omega))) \sin ^{2} 2 \theta
\end{aligned}
$$

where $p$ is the differential polarizability ratio, $\theta$ is the angle of the incident laser with respect to the nematic director, $\Omega$ is a bend angle associated the arms of a bent core molecule (this parameter was used in the paper by Zhang et al. [35] but here we set $\Omega=180^{\circ}$ as we are using a single rodlike molecule), and $\chi$ is the tilt angle of a bent core molecules arm from the molecular long axis (again, here we set $\chi=0$ as we are using a single rodlike molecule). Figure 15 shows a schematic of the model showing $\chi$ and $\Omega$.
[1] H. Zeng, O. M. Wani, P. Wasylczyk, and A. Priimagi, Lightdriven, caterpillar-inspired miniature inching robot, Macromol. Rapid Commun. 39, 1700224 (2018).

[2] S. Schuhladen, F. Preller, R. Rix, S. Petsch, R. Zentel, and H. Zappe, Iris-like tunable aperture employing liquid-crystal elastomers, Adv. Mater. 26, 7247 (2014).

[3] T. H. Ware, J. S. Biggins, A. F. Shick, M. Warner, and T. J. White, Localized soft elasticity in liquid crystal elastomers, Nat. Commun. 7, 10781 (2016).

[4] J. M. Boothby, H. Kim, and T. H. Ware, Shape changes in chemoresponsive liquid crystal elastomers, Sens. Actuators B Chem. 240, 511 (2017).

[5] D. Mistry, S. D. Connell, S. L. Mickthwaite, P. B. Morgan, J. H. Clamp, and H. F. Gleeson, Coincident molecular auxeticity and negative order parameter in a liquid crystal elastomer, Nat. Commun. 9, 5095 (2018).

[6] R. H. Baughman, J. M. Shacklette, A. A. Zakhidov, and S. Stafström, Negative Poisson's ratios as a common feature of cubic metals, Nature (London) 392, 362 (1998).

[7] K. E. Evans and A. Alderson, Auxetic materials: Functional materials and structures from lateral thinking! Adv. Mater. 12, 617 (2000).

[8] K. K. Saxena, R. Das, and E. P. Calius, Three decades of auxetics research materials with negative Poisson's ratio: A review, Adv. Eng. Mater. 18, 1847 (2016).

[9] X. Ren, R. Das, P. Tran, T. D. Ngo, and Y. M. Xie, Auxetic metamaterials and structures: A review, Smart Mater. Struct. 27, 023001 (2018).

[10] K. Wang, Y. H. Chang, Y. Chen, C. Zhang, and B. Wang, Designable dual-material auxetic metamaterials using threedimensional printing, Mater. Des. 67, 159 (2015).

[11] Y. Suzuki, G. Cardone, D. Restrepo, P. D. Zavattieri, T. S. Baker, and T. F. Akif, Self-assembly of coherently dynamic, auxetic, two-dimensional protein crystals, Nature (London) 533, 369 (2016).

[12] R. Gatt, V. Zammit, C. Caruana, and J. N. Grima, On the atomic level deformations in the auxetic zeolite natrolite, Phys. Status Solidi B 245, 502 (2008).

[13] C. Sanchez-Valle, Z. A. D. Lethbridge, S. V. Sinogeikin, J. J. Williams, R. I. Walton, K. E. Evans, and J. D. Bass, Negative
Poisson's ratios in siliceous zeolite MFI-silicalite, J. Chem. Phys. 128, 184503 (2008).

[14] K. E. Evans, M. A. Nkansah, I. J. Hutchinson, and S. C. Rogers, Molecular network design, Nature (London) 353, 124 (1991).

[15] J. N. Grima, A. Alderson, and K. Evans, Auxetic behavior from rotating rigid units, Phys. Status Solidi B 242, 561 (2005).

[16] C. He, P. Liu, P. J. McMullan, and A. C. Griffin, Toward molecular auxetics: Main chain liquid crystalline polymers consisting of laterally attached para-quaterphenyls, Phys. Status Solidi B 242, 576 (2005).

[17] A. W. Brown and J. M. Adams, Negative Poisson's ratio and semisoft elasticity of smectic- $C$ liquid-crystal elastomers, Phys. Rev. E. 85, 011703 (2012).

[18] A. Ahsan, J. Rudnick, and R. Bruinsma, Soft elasticity of rna gels and negative Poisson ratio, Phys. Rev. E 76, 061910 (2007).

[19] T. Ting and T. Chen, Poisson's ratio for anisotropic elastic materials can have no bounds, Q. J. Mech. Appl. Math. 58, 73 (2005).

[20] J. Küpfer and H. Finkelmann, Liquid crystal elastomers: Influence of the orientational distribution of the crosslinks on the phase behavior and reorientation processes, Macromol. Chem. and Phys. 195, 1353 (1994).

[21] I. Kundler and H. Finkelmann, Strain-induced director reorientation in nematic liquid single crystal elastomers, Macromol. Rapid. Commun, 16, 679 (1995).

[22] G. C. Verwey, M. Warner, and E. M. Terentjev, Elastic instability and stripe domains in liquid crystalline elastomers, J. Phys. II. France. 6, 1273 (1996).

[23] G. R. Mitchell, F. J. Davis, and W. Guo, Strain-Induced Transitions in Liquid-Crystal Elastomers, Phys. Rev. Lett. 71, 2947 (1993).

[24] P. Bladon, E. M. Terentjev, and M. Warner, Transitions and instabilities in liquid crystal elastomers, Phys. Rev. E. 47, R3838 (1993).

[25] P. Bladon, M. Warner, and E. M. Terentjev, Orientational order in strained nematic networks, Macromol. 27, 7067 (1994).

[26] D. Mistry, P. B. Morgan, J. H. Clamp, and H. F. Gleeson, New insights into the nature of SSEity and "mechanical-Fréedericksz transitions" in liquid crystal elastomers, Soft Matter 14, 1301 (2018). 
[27] P. M. S. Roberts, G. R. Mitchell, F. J. Davis, and J. A. Pople, Mechanical switching and soft elasticity in liquid crystal elastomers, Mol. Crys. Liq. Crys. Sci. Tech. Mol. Crys. Liq. Crys. 299, 181 (1997).

[28] P. M. S. Roberts, G. R. Mitchell, and F. J. Davis, A single director switching mode for monodomain liquid crystal elastomers, J. Phys. II. France. 7, 1337 (1997).

[29] F. J. Davis and G. R. Mitchell, Liquid crystal elastomers: Controlled crosslinking in the liquid crystal phase, Polymer 37, 1345 (1996).

[30] H. Finkelmann, A. Greve, and M. Warner, The elastic anisotropy of nematic elastomers, Eur. Phys. J. E. 5, 281 (2001).

[31] M. Warner and E. M. Terentjev, Liquid Crystal Elastomers (Oxford University Press, Oxford, UK, 2007).

[32] S. M. Clarke, A. Hotta, A. R. Tajbakhsh, and E. M. Terentjev, Effect of crosslinker geometry on equilibrium thermal and mechanical properties of nematic elastomers, Phys. Rev. E. 64, 061702 (2001).

[33] K. Urayama, R. Mashita, I. Kobayashi, and T. Takigawa, Stretching-induced director rotation in thin films of liquid crystal elastomers with homeotropic alignment, Macromol. 40, 7665 (2007).

[34] D. Mistry and H. F. Gleeson, Mechanical deformations of a liquid crystal elastomer at director angles between $0^{\circ}$ and $90^{\circ}$ : Deducing an empirical model encompassing anisotropic nonlinearity, J. Polym. Sci. B Polym. Phys. 57, 1367 (2019).

[35] Z. Zhang, S. Kaur, B. Kundu, B. K. Sadashiva, and H. F. Gleeson, Observing the emergence of phase biaxiality in a polar smectic a system via polarised Raman spectroscopy, J. Mater. Chem. C. 5, 1195 (2017)

[36] C. D. Southern, P. D. Brimicombe, S. D. Siemianowski, S. Jaradat, N. Roberts, V. Görtz, J. W. Goodby, and H. F. Gleeson, Thermotropic biaxial nematic order parameters and phase transitions deduced by Raman scattering, Europhys. Lett. 82, 56001 (2008).

[37] C. D. Southern and H. F. Gleeson, Using the full Raman depolarization in the determination of the order parameters in liquid crystal systems, Eur. Phys. J. E. 24, 119 (2007).

[38] Z. Zhang and H. F. Gleeson, Understanding liquid crystal order parameters deduced from different vibrations in polarised Raman spectroscopy, Liq. Cryst. 46, 219 (2019).

[39] Z. Zhang, V. P. Panov, M. Nagaraj, R. J. Mandle, J. W. Goodby, G. R. Luckhurst, J. C. Jones, and H. F. Gleeson, Raman scattering studies of order parameters in liquid crystalline dimers exhibiting the nematic and twist-bend nematic phases, J. Mater. Chem. C. 3, 10007 (2015).

[40] G. R. Luckhurst and T. J. Sluckin, Biaxial Nematic Liquid Crystals (Wiley Online Library, The Atrium, Chichester, UK, 2015).

[41] S. Jen, N. A. Clark, P. S. Pershan, and E. B. Priestley, Polarized Raman scattering studies of orientational order in uniaxial liquid crystalline phases, J. Chem. Phys. 66, 4635 (1977).
[42] A. Sanchez-Castillo, M. A. Osipov, and F. Giesselmann, Orientational order parameters in liquid crystals: A comparative study of x-ray diffraction and polarized Raman spectroscopy results, Phys. Rev. E 81, 021707 (2010).

[43] M. Warner, P. Bladon, and E. M. Terentjev, "Soft elasticity"deformation without resistance in liquid crystal elastomers, J. Phys. II. France. 4, 93 (1994).

[44] Z. Zeng, L. Jin, and Y. Huo, Strongly anisotropic elastic moduli of nematic elastomers: Analytical expressions and nonlinear temperature dependence, Eur. Phys. J. E. 32, 71 (2010).

[45] B. J. Frisken and P. Palffy-Muhoray, Freedericksz transitions in nematic liquid crystals: The effects of an in-plane electric field, Phy. Rev. A. 40, 6099 (1989).

[46] G. R. Luckhurst and C. Zannoni, Why is the Maier-Saupe theory of nematic liquid crystals so successful? Nature (London) 267, 412 (1977).

[47] R. L. Humphries, P. G. James, and G. R. Luckhurst, Molecular field treatment of nematic liquid crystals, J. Chem. Soc. Faraday Trans. 68, 1031 (1972).

[48] H. Pottel, W. Herreman, B. W. V. der Meer, and M. Ameloot, On the significance of the fourth-rank orientational order parameter of fluorophores in membranes, Chem. Phys. 102, 37 (1986).

[49] C. Zannoni, in Polarized Spectroscopy of Ordered Systems, edited by B. Samori and E. W. Thulstrup (Springer, Dordrecht, 1988), Chap. 3, pp. 57-83.

[50] C. Chiccoli, P. Pasini, F. Biscarini, and C. Zannoni, The P 4 model and its orientational phase transition, Mol. Phys. 65, 1505 (1988).

[51] C. Chiccoli, P. Pasini, and C. Zannoni, Phase diagram and orientational order of a system with second and fourth rank interactions, Int. J. Mod. Phys. B. 11, 1937 (1997).

[52] M. V. Gurp, The use of rotation matrices in the mathematical description of molecular orientations in polymers, Colloid Polym. Sci. 273, 607 (1995).

[53] D. Rogez and P. Martinoty, Mechanical properties of monodomain nematic side-chain liquid-crystalline elastomers with homeotropic and in-plane orientation of the director, Eur. Phys. J. E 34, 69 (2011).

[54] M. Warner and S. Kutter, Uniaxial and biaxial soft deformations of nematic elastomers, Phys. Rev. E 65, 051707 (2002).

[55] See Supplemental Material at http://link.aps.org/supplemental/ 10.1103/PhysRevResearch.3.023191 for a video showing the evolution of the ODF as a function of strain.

[56] B. D. Caddock and K. E. Evans, Microporous materials with negative Poisson's ratios. I. microstructure and mechanical properties, J. Phys. D: Appl. Phys. 22, 1877 (1989).

[57] K. E. Evans and B. D. Caddock, Microporous materials with negative Poisson's ratios. ii. mechanisms and interpretation, J. Phys. D: Appl. Phys. 22, 1883 (1989).

[58] V. S. R. Jampani, R. H. Volpe, K. R. de Sousa, J. F. Machado, C. M. Yakacki, and J. P. F. Lagerwall, Liquid crystal elastomer shell actuators with negative order parameter, Sci. Adv. 5, eaaw2476 (2019).

[59] https://doi.org/10.5518/795. 